ARTICLE

Received 1 Dec 2014 | Accepted 30 Jan 2015 | Published 16 Mar 2015

DOI: $10.1038 /$ ncomms7517

\title{
Transformation of the intestinal epithelium by the MSI2 RNA-binding protein
}

\author{
Shan Wang ${ }^{1,2, *}$, Ning $\mathrm{Li}^{2, \star}$, Maryam Yousefi ${ }^{2,3}$, Angela Nakauka-Ddamba ${ }^{2}$, Fan $\mathrm{Li}^{4,5,6}$, Kimberly Parada ${ }^{2}$, \\ Shilpa Rao ${ }^{7}$, Gerard Minuesa ${ }^{8}$, Yarden Katz ${ }^{9}$, Brian D. Gregory ${ }^{4,5,6}$, Michael G. Kharas ${ }^{8}$, Zhengquan $\mathrm{Yu}^{1} \&$ \\ Christopher J. Lengner $2,3,10,11,12$
}

The MSI2 RNA-binding protein is a potent oncogene playing key roles in haematopoietic stem cell homeostasis and malignant haematopoiesis. Here we demonstrate that MSI2 is expressed in the intestinal stem cell compartment, that its expression is elevated in colorectal adenocarcinomas, and that MSI2 loss-of-function abrogates colorectal cancer cell growth. MSI2 gain-of-function in the intestinal epithelium in a drug-inducible mouse model is sufficient to phenocopy many of the morphological and molecular consequences of acute loss of the APC tumour suppressor in the intestinal epithelium in a Wnt-independent manner. Transcriptome-wide RNA-binding analysis indicates that MSI2 acts as a pleiotropic inhibitor of known intestinal tumour suppressors including Lrig1, Bmpr1a, Cdkn1a and Pten. Finally, we demonstrate that inhibition of the PDK-AKT-mTORC1 axis rescues oncogenic consequences of MSI2 induction. Taken together, our findings identify MSI2 as a central component in an unappreciated oncogenic pathway promoting intestinal transformation.

\footnotetext{
${ }^{1}$ State Key Laboratories for Agrobiotechnology, College of Biological Sciences, China Agricultural University, Beijing 100194, China. ${ }^{2}$ Department of Animal Biology, School of Veterinary Medicine, University of Pennsylvania, Philadelphia, Pennsylvania 19104, USA. ${ }^{3}$ Cell and Molecular Biology Graduate Program, University of Pennsylvania, Philadelphia, Pennsylvania 19104, USA. ${ }^{4}$ Department of Biology, School of Arts and Sciences, University of Pennsylvania, Philadelphia, Pennsylvania 19104, USA. ${ }^{5}$ PENN Genome Frontiers Institute, University of Pennsylvania, Philadelphia, Pennsylvania 19104, USA. ${ }^{6}$ Genomics and Computational Biology Graduate Program, University of Pennsylvania, Philadelphia, Pennsylvania 19104, USA. ${ }^{7}$ PENN Molecular Profiling Facility, University of Pennsylvania, Philadelphia, Pennsylvania 19104, USA. ${ }^{8}$ Molecular Pharmacology and Chemistry Program, Experimental Therapeutics Center and Center for Stem Cell Biology, Memorial Sloan-Kettering Cancer Center, New York, New York 10065, USA. ${ }^{9}$ The Broad Institute of Harvard and MIT, 415 Main Street, Cambridge, Massachusetts 02142, USA. ${ }^{10}$ Center for Molecular Studies in Digestive and Liver Diseases, University of Pennsylvania, Philadelphia, Pennsylvania 19104, USA. ${ }^{11}$ Department of Cell and Developmental Biology, School of Medicine, University of Pennsylvania, Philadelphia, Pennsylvania 19104 USA. ${ }^{12}$ Institute for Regenerative Medicine, University of Pennsylvania, Philadelphia, Pennsylvania 19104, USA. * These authors contributed equally to this work. Correspondence and requests for materials should be addressed to Z.Y. (email: Zyu@cau.edu.cn) or to C.J.L. (email: Lengner@vet.upenn.edu).
} 
T he Drosophila melanogaster RNA-binding protein Musashi contributes to asymmetric stem cell division and cell fate determination in the neuroblast ${ }^{1}$. In mammals, there are two Musashi orthologues, MSI1/Msi1 and MSI2/Msi2 (ref. 2,3). Recently, Msi2 has been implicated as a critical regulator of haematopoietic stem cell (HSC) self-renewal and fate determination and MSI2 is a potent cooperative oncogene in human leukaemias ${ }^{4-6}$. The role of MSI2 in leukaemia progression was recently revealed by two groups who independently observed increased MSI2 expression during disease progression in patients with chronic myelogenous leukemia (CML) blast crisis and in acute myeloid leukaemias ${ }^{4,5}$. Forced MSI2 expression drove a more aggressive myeloid disease in a transplantation model utilizing the BCR-ABL oncogene. In contrast, MSI2 abrogation in myeloid leukaemia cells increases differentiation, decreases proliferation and increases apoptosis ${ }^{4}$. These studies demonstrate that MSI2 cooperates with known oncogenes in haematopoietic malignancies. In addition, high MSI2 expression is observed in a variety of other cancers, including hepatocellular carcinoma and lung cancer ${ }^{7,8}$, suggesting an important role for MSI2 in a variety of epithelial-derived carcinomas.

Aggressive leukaemias are characterized by the prevalence of an increasingly HSC-like transcriptional profile. Consistent to the role of MSI2 in leukaemia, MSI2 also plays an important role in HSC homeostasis. MSI2 is highly expressed in the most primitive HSCs, including long-term HSCs and short-term HSCs, but not in more committed haematopoietic lineages. Inactivation of Msi2 in HSCs impairs their competitive repopulation ability upon transplantation ${ }^{4,6,9}$. Thus, although the functions of MSI2 in normal and malignant haematopoiesis are well established, little is known regarding the role MSI2 plays in stem cells and cancers in other organ systems.

In contrast to reports of MSI2 function in the haematopoietic system, several reports have suggested a role for the second Musashi family member, MSI1 in colorectal cancer (CRC). Msil is expressed in the putative intestinal stem cell (ISC) compartment ${ }^{10}$ and overexpressed in colorectal adenocarcinoma, where higher expression level of MSI1 is correlated to increased metastatic risk and poorer survival ${ }^{11,12}$. The putative role of MSI1 in CRC and ISCs, coupled with our past observations of MSI2 function in the HSC and haematopoietic cancers, prompted us to investigate a role of MSI2 in intestinal transformation.

$\mathrm{CRC}$ is one the leading causes of cancer-related deaths globally. Genetic inactivation of the adenomatous polyposis coli (APC) tumour suppressor gene is believed to initiate the majority of human CRCs, and elegant genetic studies suggest that APC loss only initiates tumorigenesis when it occurs in ISCs with selfrenewal capacity ${ }^{13}$. APC loss drives constitutive activity of the canonical Wnt signalling pathway by preventing the degradation of its downstream transcriptional effector $\beta$-catenin. Thus, constitutive $\beta$-catenin activity is thought to be a primary initiator of ISC transformation. Genetic inactivation of $A P C$ is found in $\sim 80 \%$ of human patients with CRC, and families harbouring a germline mutation in one APC allele suffer from familial adenomatous polyposis, a disease characterized by the formation of numerous intestinal polyps resulting from stochastic loss of heterozygosity (LOH) at the APC locus, some of which will invariably progress to $\mathrm{CRC}^{14-16}$. The role of MSI2 in this process and its potential interaction with the Wnt signalling pathway remains entirely unknown.

In this study, we find that MSI2 is overexpressed in human colorectal adenocarcinomas, as well as in early-stage adenomas arising in the $A P C^{\mathrm{min} /+}$ mouse model of intestinal tumorigenesis. Using both loss- and gain-of-function approaches we demonstrate that constitutive MSI2 activation is sufficient to phenocopy many histological and molecular aspects of APC loss in the absence of canonical Wnt pathway induction. Transcriptome-wide RNA-binding analysis reveals that MSI2 binds several known intestinal tumor suppressor transcripts, including Pten, a potent tumour suppressor frequently silenced in CRCs. Our findings identify MSI2 as an intestinal oncogene and suggest that MSI2 inhibition may offer a novel point for therapeutic intervention in gastrointestinal (GI) cancers.

\section{Results}

MSI2 is overexpressed in intestinal cancers. To begin understanding the role of MSI2 in CRC, we examined MSI2 expression in human GI cancers, and colorectal adenocarcinomas in particular. Analysis of transcriptome profiles from a number of GI cancers reveals elevated MSI2 in all analysed tumours, including adenocarcinomas of the caecum, colon and rectum (Supplementary Fig. 1a). Focusing on colorectal adenocarcinoma, analysis of cancer genome atlas data (TCGA) derived form 314 healthy control individuals and 314 colon adenocarcinomas patient tumours revealed consistent MSI2 overexpression in cancer tissue (Fig. 1a). Similarly, analysis of 26 colon adenocarcinoma/adjacent normal tissue pairs from individual patients revealed uniform overexpression of MSI2 in tumours (Fig. 1b). Strikingly, MSI2 overexpression was more consistent in these matched samples than that of the well-established colorectal oncogene $c-M Y C$, which was underexpressed in a small fraction of these tumours (Fig. 1b). Consistent with the upregulation of MSI2 transcripts, immunohistochemistry analysis of a CRC tissue array confirmed high MSI2 immunoreactivity in all grades of CRC, with an increased MSI2-immunoreactive area correlating with the tumour grade, suggesting that MSI2 activation may be an early event in the ontogeny of cancer (Fig. 1c and Supplementary Fig. 1b). We also examined Msi2 expression in intestinal adenomas resulting from $\mathrm{LOH}$ of the APC tumour suppressor in the $A P C^{\min /+}$ mouse model of intestinal transformation. APC loss occurs in the vast majority of human CRCs, where it is believed to be the initiating oncogenic event. Consistent with our observation in human tumours, adenomas in $A P C^{\text {min/+ }}$ mice exhibited strong Msi2 immunoreactivity, suggesting a potential role for Msi2 early in intestinal transformation and tumorigenesis (Fig. 1d). In order to determine whether Msi2 activation was an immediate early event downstream of APC loss, we deleted APC throughout the epithelium using Villin-CreER $R^{17}$ and observed a striking upregulation of Msi2 mRNA along with the activation of Wnt target genes (Axin2, Ascl2 and Lgr5) and suppression of differentiation markers Chromogranin-A and Lysozyme characteristic of acute APC loss (ref. 18; Fig. 1e). Thus, Msi2 activation is a direct consequence of APC loss and is characteristic of all grades of colorectal adenocarcinoma.

MSI2 promotes cancer cell proliferation and tumour growth. To investigate a potential oncogenic role for MSI2 in CRC, we initially performed MSI2 loss-of-function experiments in two human CRC cell lines, SW48 and HT29. Numerous studies have implicated constitutive Wnt pathway activity as a driving force in CRC onset and progression, and these cancers are dependent on the transcription activity of the Wnt effector $\beta$-catenin ${ }^{19}$. We therefore knocked down MSI2 alone or in combination with $\beta$-catenin and confirmed loss of MSI2 protein using western blot analysis (Supplementary Fig. 1c). Surprisingly, MSI2 knockdown completely abrogated growth of SW48 and HT29 cells, and was as potent as loss of $\beta$-catenin in growth inhibition (Fig. 1f,g). This finding strongly indicates an important role for MSI2 in promoting cancer cell proliferation. We confirmed these in vitro observations by performing xenograft assays on MSI2 
a

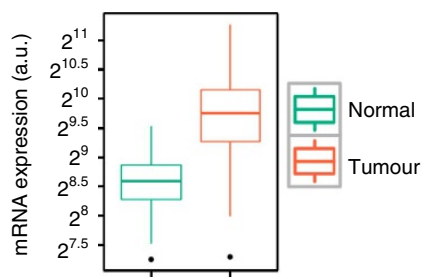

b

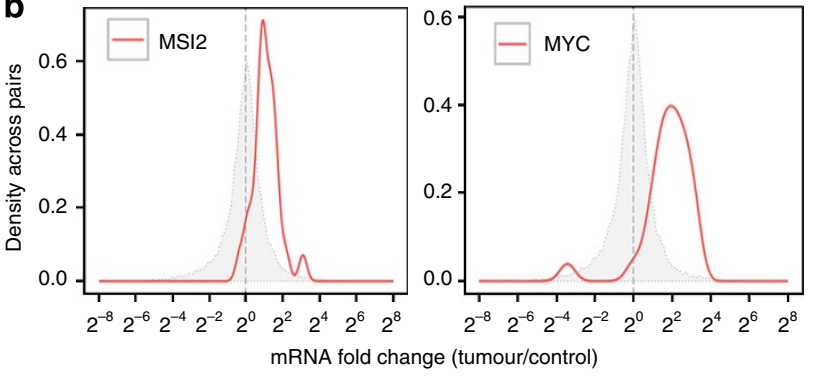

d
C
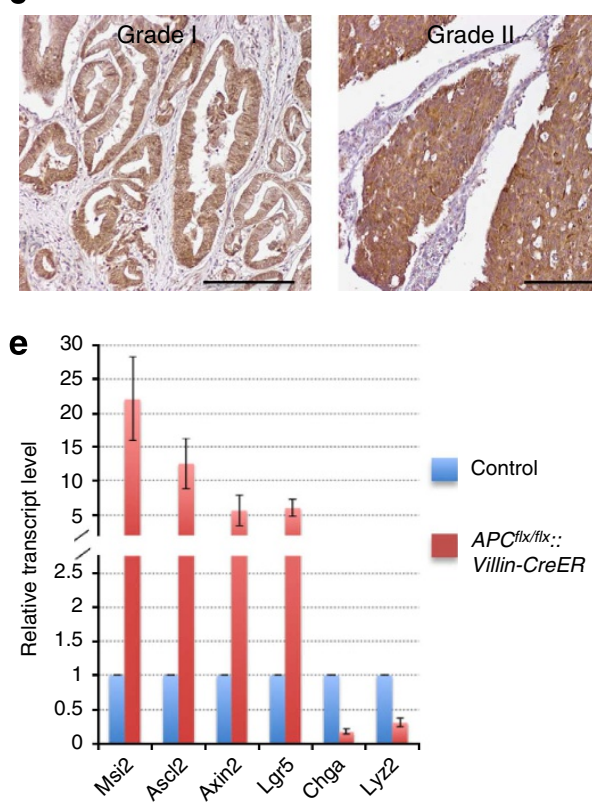

g

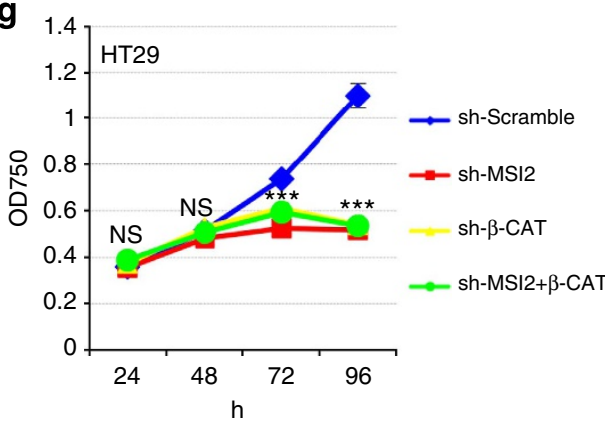

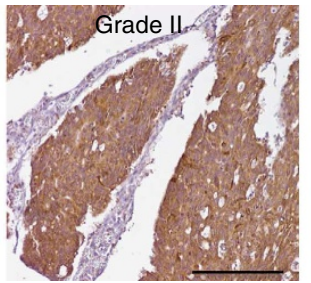
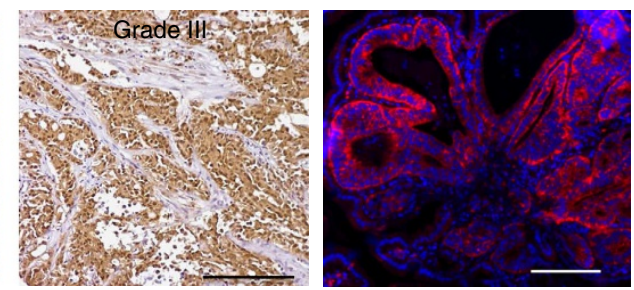

f

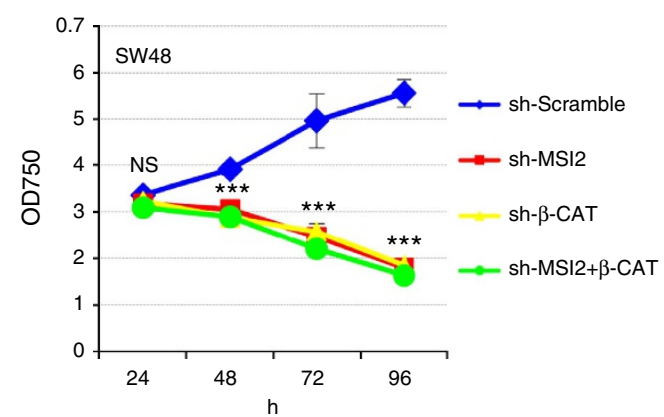

h

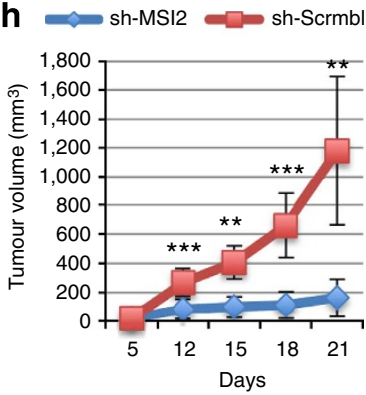

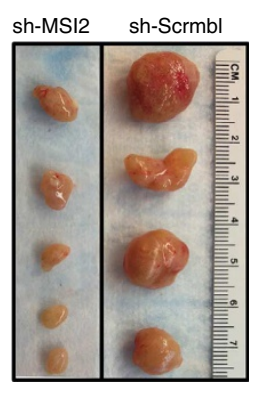

Figure 1 | MSI2 contributes to human colorectal cancer cell growth. (a) MSI2 mRNA expression levels from RNA-Seg on TCGA (the cancer genome atlas) COAD (colorectal adenocarcinoma) tumour samples (314 solid tissue samples from healthy individuals and 314 tumour samples). Expression values were obtained by TMM normalization of read counts in genes. (b) Fold changes for MSI2 (left) and MYC (right) in matched tumour/control RNA-Seq sample pairs from TCGA COAD (total of 26 patients). The distribution of MSI2/MYC fold changes in tumour/control pairs for 26 individuals is plotted in red (intra-individual comparison). The distribution of MSI2/MYC fold changes between control/control comparisons for 26 pairs of healthy individuals is plotted in grey (interindividual comparison). (c) MSI2 immunochemistry of graded human colorectal adenocarcinomas (scale bar, $200 \mu \mathrm{M}$ ).

(d) Msi2 immunofluorescence showing broad expression in adenomas arising in APC min/ + mice (scale bar, 200 $\mu$ M). (e) Expression analysis for Msi2, Wnt target genes (Ascl2, Axin2 and Lgr5) and differentiation-related genes (Chga and Lyz) in the intestinal epithelium after acute ablation of APC in $A P C^{f l o x} /$ flox::Villin-CreER mice (error bars denotes s.d., $\left.n=3\right) .(\mathbf{f}, \mathbf{g})$ MTT proliferation assays of human colorectal cancer cell lines SW48 (f) and HT29 (g) infected with lentiviral knockdown vectors for MSI2, $\beta$-catenin or scramble controls. Error bars indicated s.e.m. from six independent experiments per cell line per condition. ${ }^{\star \star \star} P<0.0005$, Student's $t$-test. (h) Tumour growth curves of HT29 colon cancer cell xenografts infected with lentivirus expressing scramble control hairpin or sh-MSI2. Error bars denote the s.d. derived from four tumours in each of two mice per group. Representative images of xenograft tumours are shown on the right. ${ }^{\star \star} P<0.005$, ${ }^{\star \star \star} P<0.0005$, Student's $t$-test.

knockdown in HT29 cells. Twenty-one days after xenografting, tumour volume was significantly reduced in MSI2 knockdown tumours, with some xenografts exhibiting no growth after transplantation (Fig. 1h).
MSI2 overexpression largely phenocopies APC loss. To gain insight into the potential function of Msi2 in intestinal homeostasis and transformation, we examined Msi2 expression in wild-type mice and found it to be largely restricted to cells of the 
crypt in the small intestine. This includes crypt base columnar (CBC) stem cells marked by expression of the Wnt co-receptor Lgr5 (ref. 20), Paneth cells and the rapidly proliferating cells of the transit-amplifying zone (Fig. 2a,b, and Supplementary Fig. 2a). In addition, rare Msi2-positive cells were observed in the differentiated villi, possibly marking enteroendocrine or tuft cells on the basis of frequency and position (Supplementary Fig. 2a). We next sought to determine the functional consequences of ectopic MSI2 activity in the intestinal epithelium. We therefore employed a Doxycycline (Dox)-inducible, site-specific, single-copy human MSI2 transgenic mouse model (TRE-MSI2) that we previously utilized to study the oncogenic functions of MSI2 in the haematopoietic system (Supplementary
Fig. 2a) $)^{4}$. Addition of Dox to the drinking water of TRE-MSI2 mice resulted in activation of MSI2 expression throughout the intestinal epithelium within $12 \mathrm{~h}$ (Fig. $2 \mathrm{~b}$ and Supplementary Fig. 2c). Strikingly, MSI2 induction resulted in a dramatic expansion of the proliferative zone and an increased frequency of apoptotic cells at the tips of villi, suggesting that cells were being pushed from the crypt to the villus and shed into the lumen at an increased rate (Fig. 2c,d). We further observed that differentiated cells of the intestinal epithelium including goblet, enteroendocrine and enterocyte cells were obviously absent in the TRE-MSI2 mice (Fig. 2e,f and Supplementary Fig. 2d). However, lysozyme-positive Paneth cells persisted likely due to their long lifespan and stable positioning at the base of the crypt
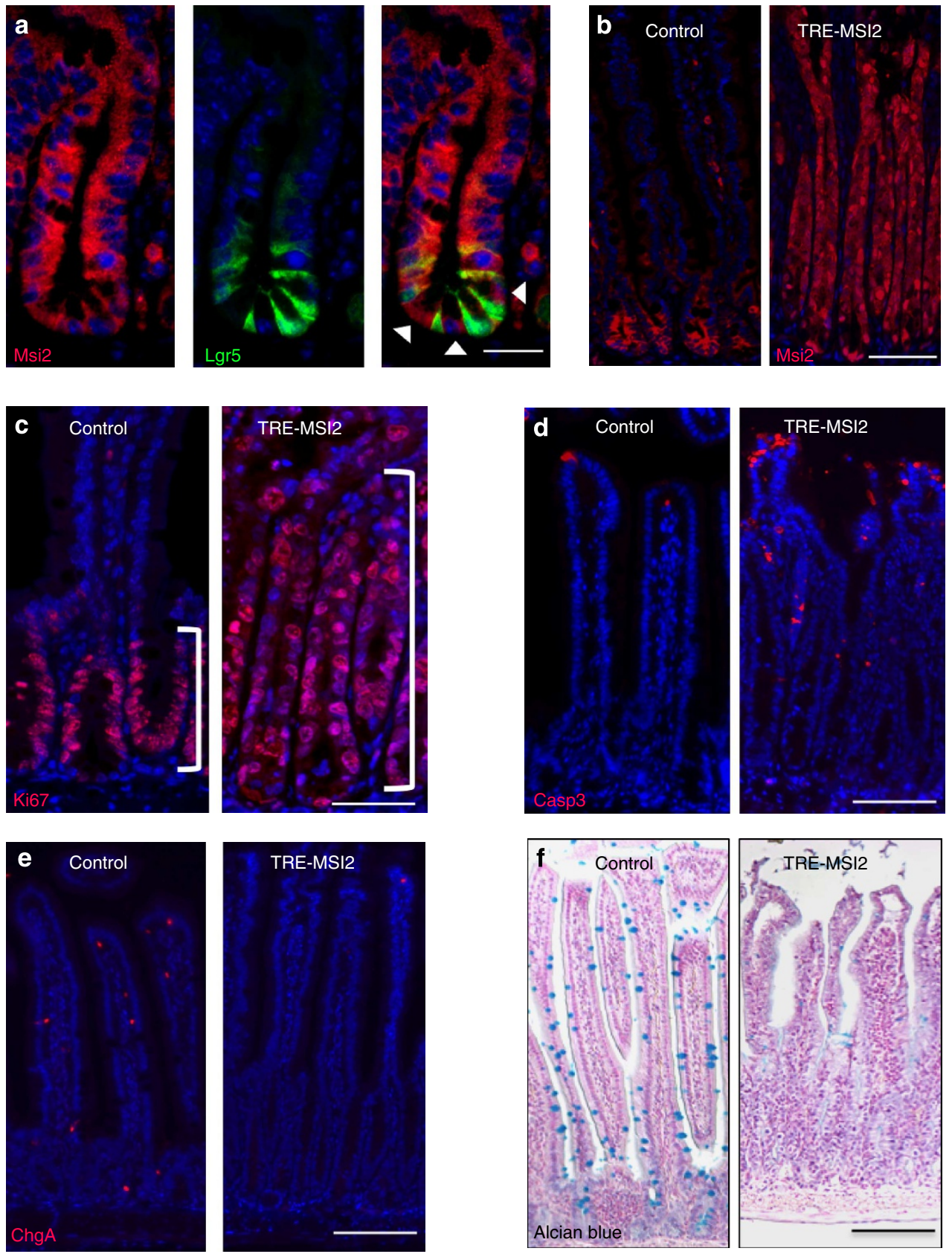

Figure 2 | MSI2 induction drives intestinal epithelial cell hyperproliferation and blocks differentiation. (a) Immunofluorescence for Msi2 (red) and eGFP (green) in the intestinal crypts of Lgr5-eGFP-IRES-CreER knock-in mice. Msi2 expression is observed in Lgr5 + cells and in adjacent Paneth cells (arrowheads; scale bar, $50 \mu \mathrm{M}$ ). (b-f) Immunofluorescence for MSI2 (b), Ki67 marking proliferating cells (c), cleaved Caspase 3 (Casp3) marking apoptotic cells in d, Chromagranin-A marking enteroendocrine cells (e) and Alcian blue marking Goblet cells (f) in control (M2rtTA) and TRE-MSI2 intestines $48 \mathrm{~h}$ after $2 \mathrm{mg} \mathrm{ml}^{-1}$ Dox induction. Brackets in $\mathbf{c}$ indicate the crypt-proliferative zone. Scale bars in $\mathbf{b}-\mathbf{f}, 100 \mu \mathrm{M}$. 
(Supplementary Fig. 2e). This block in differentiation resulted in severe dehydration and morbidity within 3-4 days of Dox induction.

To further investigate the effect of MSI2 induction on proliferation and the crypt stem/progenitor cell compartment, we quantified crypt height and crypt fission (frequency of crypts per linear unit) in TRE-MSI2 mice and observed a significant increase in both of these parameters (Fig. 3a,b). Interestingly, the increased crypt proliferation, crypt fission, block in differentiation and rapid morbidity in TRE-MSI2 mice are all phenotypes entirely consistent with the consequences downstream of acute APC loss in the intestinal epithelium ${ }^{18,21}$. Taken together, these observations suggest that MSI2 induction drives proliferative expansion of an undifferentiated stem/progenitor cell and may act downstream of APC loss to promote intestinal transformation.

APC loss drives intestinal tumorigenesis only when it occurs in an ISC $^{13}$. We therefore sought to assess the effects of MSI2 induction specifically in the ISC compartment. We bred TREMSI2 mice to Lgr5-eGFP-IRES-CreER reporter mice that mark CBC stem cells ${ }^{20}$ and observed a dramatic expansion of the Lgr5 ${ }^{+}$zone within the crypts of TRE-MSI2 mice (Fig. 3c). This expansion was accompanied by an overall increase in the proliferation rate of intestinal epithelial cells (Fig. 3d,e), as well as an increase in the absolute frequency and proliferation rate of Lgr5 ${ }^{+}$CBCs in TRE-MSI2 mice relative to controls (Fig. 3f,g).
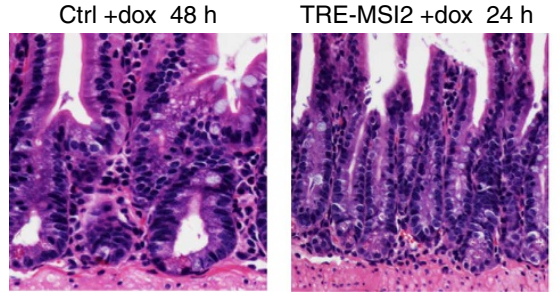

b
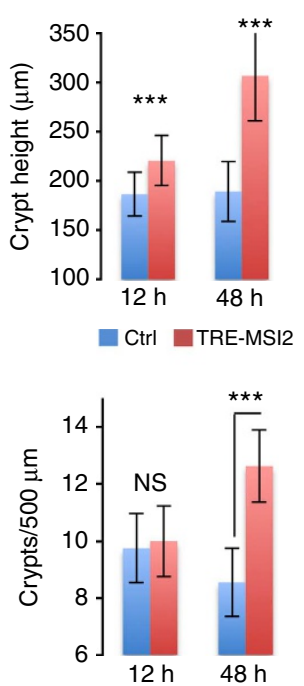

d

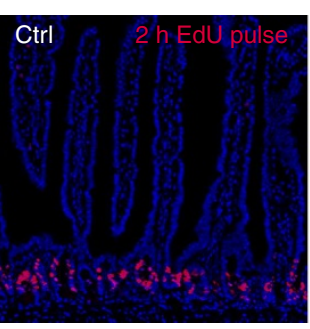

e

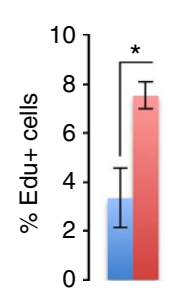

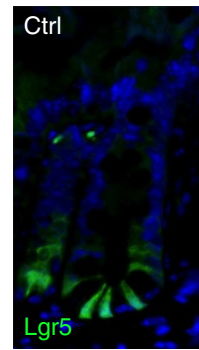
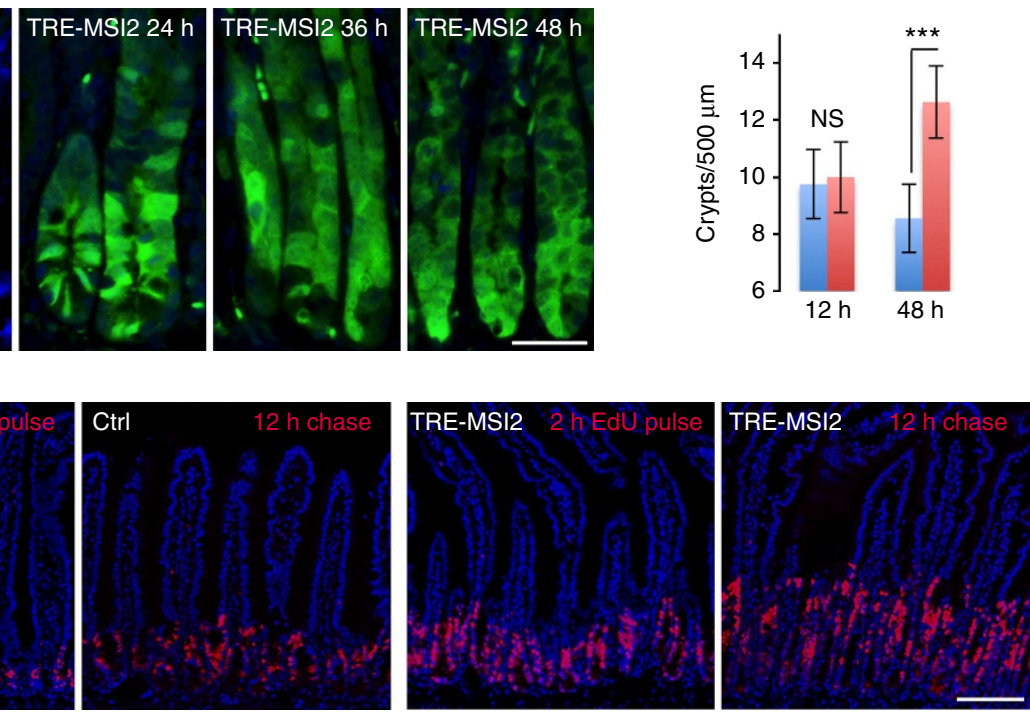

h
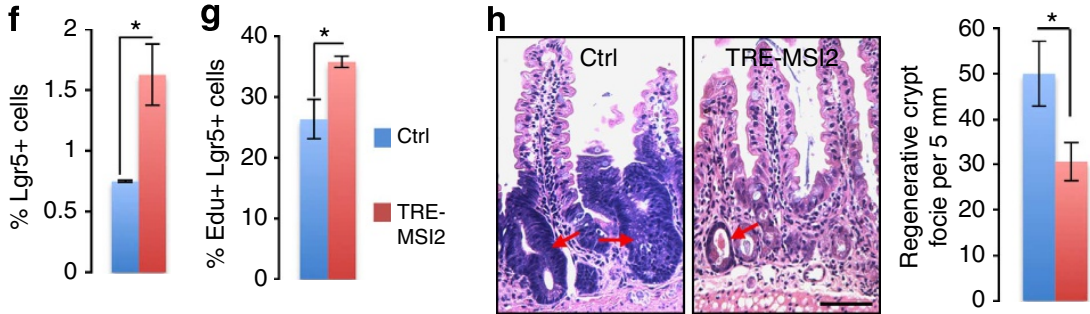

Figure 3 | MSI2 expression induces crypt fission and expansion of intestinal stem cells. (a) Haematoxylin and eosin staining of control (M2rtTA) and TRE-MSI2 small intestine treated with $2 \mathrm{mg} \mathrm{ml}^{-1}$ Dox for 24 and $48 \mathrm{~h}$ showing expanded crypt height and increased crypt fission (scale bar, $100 \mu \mathrm{M}$ ). (b) Quantification of crypt length (top panel) and crypt fission (lower panel) in control (M2rtTA) and TRE-MSI2 intestines at indicated time points after the Dox treatment. Error bars indicate the s.d. derived from $n=3$ mice per group per time point. ${ }^{\star \star \star} P<0.0005$, Student's $t$-test. (c) Immunofluorescence for eGFP in control (Lgr5-eGFP-IRES-CreER::M2rtTA) and TRE-MSI2::Lgr5-eGFP-IRES-CreER intestinal crypts at the indicated time points after Dox induction $($ scale bar, $50 \mu \mathrm{M})$. (d) Histological staining for EdU incorporation 2 or $12 \mathrm{~h}$ after an EdU pulse in control (M2rtTA) and TRE-MSI2 mice. Images were taken $48 \mathrm{~h}$ after initiation of $2 \mathrm{mg} \mathrm{ml}^{-1}$ Dox treatment (scale bar, $100 \mu \mathrm{M}$ ). (e) Flow cytometric analysis of EdU incorporation in control (M2rtTA) and TRE-MSI2 mice $48 \mathrm{~h}$ after $2 \mathrm{mg} \mathrm{ml}^{-1}$ Dox induction. Error bars denote s.d. ( $n=3$ mice per group), ${ }^{\star} P<0.05$, Student's $t$-test. (f,g) Flow cytometric analysis of Lgr5-eGFP + cell frequency (f) and 2-h-EdU incorporation in Lgr5-eGFP + cells (g) from control (Lgr5-eGFP-IRES-CreER::M2rtTA) and TRE-MSI2::Lgr5-eGFPIRES-CreER mice $48 \mathrm{~h}$ after $2 \mathrm{mg} \mathrm{ml}^{-1}$ Dox treatment. Error bars denote s.d. ( $n=3$ mice per group), ${ }^{*}<0.05$, Student's $t$-test. (h) Histological analysis and quantification of regenerative crypt foci (arrows) in control (Msi2 ${ }^{\text {wt } / w t}::$ Villin-CreER) or Msi2 flox/flox::Villin-CreER mice 3 days after exposure to $12-G y$ gamma irradiation (scale bar, $50 \mu \mathrm{M}$ ). Error bars denote s.d. ( $n=3$ mice per group). ${ }^{\star} P<0.05$, Student's $t$-test. 
To test whether the increased apoptotic events at the villus tip in TRE-MSI2 might be a result of increased turnover of the epithelium and more rapid replacement of the differentiated villus compartment with proliferative cells from the crypts, we assessed the migration of ethynyl-2'-deoxyuridine (EdU)-labelled cells after $12 \mathrm{~h}$ in the presence of ectopic MSI2 and found that, indeed, proliferative cells in TRE-MSI2 mice migrated higher than their control counterparts (Fig. 3d). Taken together, these findings are consistent with a model where MSI2 increases the shedding of differentiated cells and promotes their replacement by undifferentiated cells.

To determine whether the effects of MSI2 induction are epithelial cell-autonomous, we cultured crypts from TREMSI2::Lgr5-eGFP-IRES-CreER in vitro and observed an increase in crypt budding and Lgr5 ${ }^{+}$cell expansion, consistent with an epithelial cell-autonomous phenotype (Supplementary Fig. 3a,b). Thus, MSI2 induction drives the proliferative expansion of undifferentiated ISCs. This suggests that endogenous Msi2 may play a role in intestinal epithelial proliferation and regeneration. To test this, we first examined regenerative crypt foci 3 days after exposure of wild-type mice to high-dose (12 Gy) gamma irradiation and observed high Msi2 expression in proliferative cells (Supplementary Fig. 3c). We next ablated Msi2 expression throughout the intestinal epithelium using Msifflox/flox::VillinCreER mice ${ }^{6}$ and observed that in the absence of Msi2 activity there was a significant decrease in the formation of regenerative crypt foci (Fig. $3 \mathrm{~h}$ ), indicating that endogenous Msi2 plays a role in supporting proliferation in the epithelium.

MSI2-driven transformation is Msil-independent. Msi2/MSI2 is one of two mammalian orthologues of D. melanogaster Musashi. The second mammalian protein, Msil/MSI1, is expressed in ISCs and has been implicated as an oncogene in intestinal cancers and elsewhere ${ }^{11,22-24}$. We therefore examined Msi1 expression in the TRE-MSI2 intestinal epithelium and observed a striking activation of Msil throughout the epithelium in response to MSI2 induction (Fig. 4a-c). RNA analysis demonstrates that the activation of Msi1 in response to MSI2 induction occurs at the transcript level, and that the activation is not reciprocal: Msi1 induction in an analogous mouse model (TRE-Msi1) did not result in Msi2 activation (Fig. 4b).

We therefore tested whether the phenotype resulting from MSI2 induction was Msil-dependent. We generated Msi $1^{\text {flox/flox:: }}$ Villin-CreER mice ${ }^{25}$ enabling the deletion of Msil within all cells of the intestinal epithelium, and deleted Msi1, and induced MSI2 with Dox. Msi1 deletion with Villin-CreER resulted in no morphological phenotype during homeostasis consistent with the viability and lack of any overt GI phenotype in germline Msi1 knockout mice ${ }^{26}$. Deletion of Msi1 was confirmed at the protein level (Fig. 4c,e). We observed that the phenotype resulting from MSI2 induction was fully penetrant in the absence of Msi1, with increased crypt proliferation and fission, and decreased differentiation being unaffected in TRE-MSI2::Msif flox/flox::VillinCreER mice (Fig. 4d-f and Supplementary Fig. 3d). Thus, Msi1 activity is not required for MSI2-driven transformation of the intestinal epithelium.

The oncogenic effects of MSI2 are $\beta$-catenin-independent. To gain mechanistic insights into the molecular events underlying the effects of MSI2 induction, we analysed transcriptome profiles of intestinal epithelia $24 \mathrm{~h}$ after Dox administration to TRE-MSI2 or control (M2rtTA) mice, before overt phenotypic changes. MSI2 induction drove rapid and robust changes in the intestinal epithelial transcriptome (Fig. 5a and Supplementary Table I). Unbiased gene set enrichment analysis (GSEA) ${ }^{27}$ of these changes revealed that the APC-loss gene signature was among the most significantly enriched in the TRE-MSI2 epithelium (Fig. 5b and Supplementary Fig. 4a,b). Thus, genes that are activated upon APC loss are also activated upon MSI2 induction, and genes suppressed upon APC loss are similarly suppressed upon MSI2 induction, with a false discovery rate (FDR) approaching zero.

In addition to the APC loss gene signature, MSI2 induction significantly enriched gene signatures associated with RNA processing, RAS oncogenic signalling and a common stem cell gene signature, suggesting that MSI2 drives proliferative expansion of an undifferentiated stem/progenitor cell, consistent with the observed increase in $\operatorname{Lgr} 5^{+}$cell frequency and cycling (Supplementary Fig. 4c). Further, MSI2 activity strongly suppressed an oxidative phosphorylation expression signature, indicating that a metabolic shift consistent with the Warburg effect may occur in response to MSI2 induction (Supplementary Fig. 4c).

Given the ability of MSI2 induction to phenocopy many aspects of APC at both the molecular and morphological level, we tested whether MSI2 activation resulted in loss of APC protein, as MSI proteins are known to function as translational repressors ${ }^{3}$. We were unable to detect any decrease in APC upon MSI2 induction (Fig. 5c and Supplementary Fig. 4d). Since APC loss is believed to drive transformation of the intestinal epithelium primarily through constitutive activation of the Wnt effector $\beta$-catenin and, subsequently, hyperactivation of the canonical Wnt pathway, it was important to address the consequences of MSI2 induction for transcriptionally active (nuclear) $\beta$-catenin in TRE-MSI2 crypts. Normally, nuclear $\beta$-catenin is limited to the Lgr5 ${ }^{+}$CBCs and Paneth cells, rapidly disappearing above the +4 position of the crypt (Fig. $5 \mathrm{~d}$ ). We observed no change in the pattern or frequency of nuclear $\beta$-catenin after MSI 2 induction (Fig. 5d), or in the expression levels of known direct $\beta$-catenin target genes in the transcriptome profiles (Supplementary Fig. 4e). We also observed that canonical Wnt target genes such as Ascl2, Axin2, Lgr5 and Sox9 were not among the genes commonly upregulated on APC loss and MSI2 induction in the GSEA (Supplementary Fig. 4b).

To further explore the effect of MSI2 on activity of the canonical Wnt pathway, we analysed the expression of Wnt target genes Ascl2, Axin2, Lgr5 and Sox9, as well as differentiation markers Lysozyme and Chromogranin-A on MSI2 induction both in crypts in vivo and in organoid cultures. MSI2 had identical effects on the expression of these genes both in vivo and in vitro. Remarkably, not only were Wnt target genes Ascl2, Axin2 and Sox9 not activated, they exhibited suppression on MSI2 induction, providing further evidence that the effects of MSI2 are not mediated through canonical Wnt signalling (Fig. 5e and Supplementary Fig. 3e). One exception was Lgr5, which was activated by MSI2. Differentiation markers Lysozyme and Chromogranin- $A$ were suppressed on MSI2 induction in vivo and in vitro, consistent with the effect of APC loss on differentiation (Fig. 1e), and with the effects of MSI2 being epithelial cell-autonomous. Further, in vitro analysis of $\beta$-Catenin/TCR reporter activity (TOPFlash) confirmed that MSI2 does not activate canonical Wnt signalling (Fig. 5f).

We also directly compared the effects of $\beta$-Catenin transcriptional activation to MSI2 induction in intestinal organoid cultures from TRE-BcatS33 mice ${ }^{28}$. In these mice, a nondegradable, transcriptionally active $\beta$-Catenin is targeted to the identical chromosomal location as MSI2 in the TRE-MSI2 mouse model in the same genetic background, enabling direct comparison of the effects of these two proteins. As expected, induction of $\beta$-CateninS33 resulted in the growth of large, spherical, cystic organoids consistent with the known effects of Wnt pathway hyperactivation (Supplementary Fig. 3f). In contrast, MSI2 
a
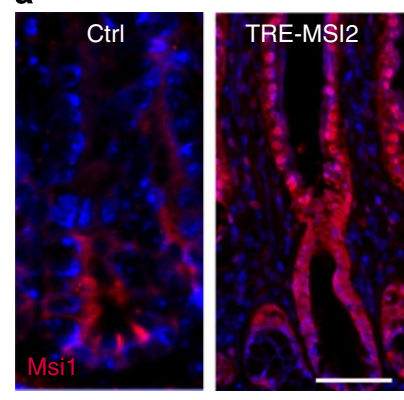

d

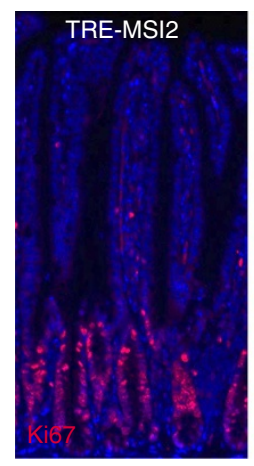

f

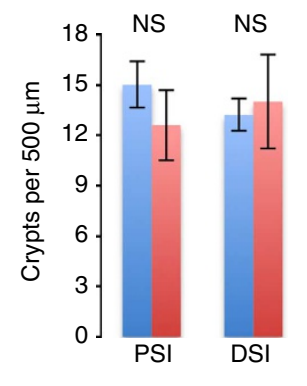

b

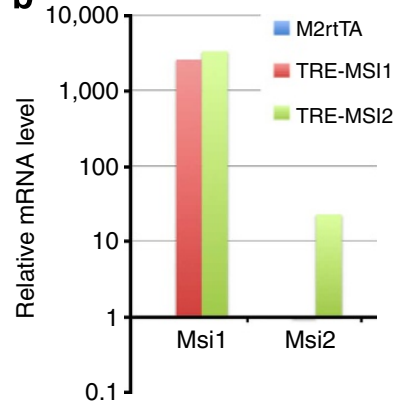

e
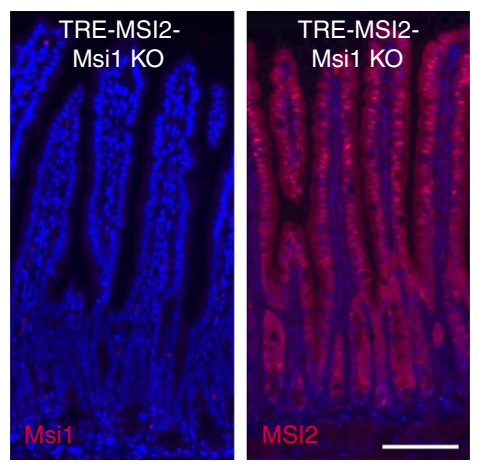

TRE-MSI2

TRE-MSI2-

Msi1 KO

Figure 4 | Msi1 is dispensable for MSI2-driven intestinal transformation. (a) Immunofluorescence for Msi1 in control (M2rtTA) and TRE-MSI2 mice (scale bar, $50 \mu \mathrm{M}$ ). (b) Quantitative RT-PCR for Msi1 and Msi2 in control (M2rtTA), TRE-Msi1 or TRE-MSI2 mice treated with $2 \mathrm{mg} \mathrm{ml}^{-1}$ Dox for $24 \mathrm{~h}$. Msi1 is induced in TRE-MSI2 mice, whereas Msi2 expression is not reciprocally effected by TRE-Msi1 induction. (c) Western blot showing Msi1 protein induction on dox treatment of TRE-MSI2 mice. Msi1 induction is prevented in TRE-MSI2::Msifflox/flox:: VillinCreER mice (TRE-MSI2-Msi7KO) after

Tamoxifen-mediated inactivation of Msi1 alleles followed by the Dox treatment. (d) Immunostaining for Ki67 in the intestinal crypts of TRE-MSI2 and TRE-MSI2::MsiTKO mice (scale bar, $100 \mu \mathrm{M}$ ). (e) Immunofluorescence for Msi1 (left) and Msi2/MSI2 (right) in TRE-MSI2-MsiTKO intestine (scale bar, $100 \mu \mathrm{M})$. (f, $\mathbf{g}$ ) Quantification of crypt fission (f) and crypt height (g) in TRE-MSI2 and TRE-MSI2::MsiTKO mice. Error bars indicate s.d. derived from at least 20 fields per slide from each of two mice per condition. PSI/DSI: proximal and distal small intestine.

induction resulted in large organoids with prolific crypt budding (Supplementary Fig. 3f), consistent with the in vivo phenotype.

Consistent with our earlier observation of elevated Msi2 expression in adenomas resulting from $\mathrm{LOH}$ in $A P C^{\mathrm{min} /+}$ mice and upon acute $A P C$ deletion in $A P C^{\text {flox/flox }}$ mice, these findings support a model in which MSI2 acts downstream of APC loss in a pathway parallel to and independent of $\beta$-catenin activation. Thus, MSI2 may cooperate with Wnt/ $\beta$-catenin signalling to drive tumorigenesis. We therefore crossed TRE-MSI2 mice with $A P C^{\mathrm{min} /+}$ mice and administered a low dose of Dox for 3 weeks $\left(0.1 \mathrm{mg} \mathrm{ml}^{-1}\right.$, or $5 \%$ of the standard dose). TRE-MSI2::APC ${ }^{\mathrm{min} /+}$ mice treated with low-dose Dox exhibited an increase in tumour burden resulting from $A P C \mathrm{LOH}$, while adjacent tissue that had not undergone $\mathrm{LOH}$ remained phenotypically normal (Fig. $5 \mathrm{~g}$ and Supplementary Fig. 4f). This finding provides additional support to the model in which MSI2 promotes tumorigenesis in a pathway parallel to canonical Wnt signalling.
Transcriptome-wide MSI2 RNA-binding analysis. MSI2 is an RNA-binding protein that, similar to MSI1, harbours two RNA recognition motifs that mediate RNA binding ${ }^{3}$. Binding of RNA targets by MSI1 results in their translational suppression without affecting overall mRNA stability ${ }^{29,30}$. Therefore, if we assume that MSI2 (which is highly homologous to MSI1, particularly in the RNA recognition motifs ${ }^{3}$ ) acts in a manner similar to MSI1, we expect that the transcriptional changes associated with MSI2 induction are not direct effects of its RNA-binding activity. To identify direct MSI2-binding targets within the intestinal epithelium, we performed crosslinking and immunoprecipitation of Msi2/MSI2-bound RNA in intestinal crypts isolated from wildtype mice and in the TRE-MSI2-transformed intestinal epithelium, followed by RNase digestion of unprotected RNA and massively parallel sequencing (RNA crosslinking, immunoprecipitation and massively parallel sequencing (CLIP-Seq)). Msi2/MSI2 primarily bound sites in $3^{\prime}$ untranslated regions (UTRs), consistent with the established binding activity of Msi1 
a

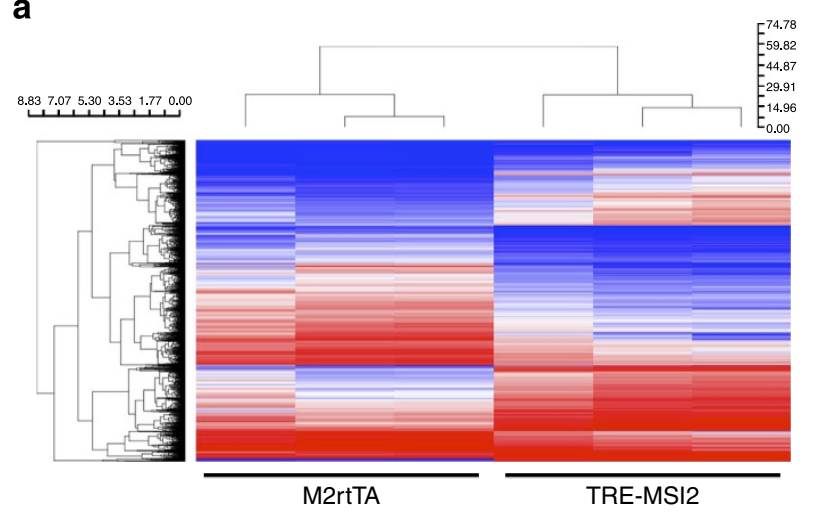

b

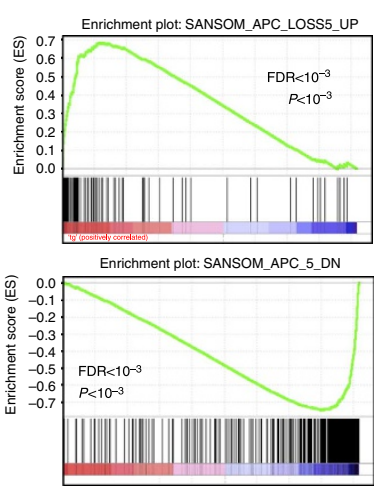

C

d
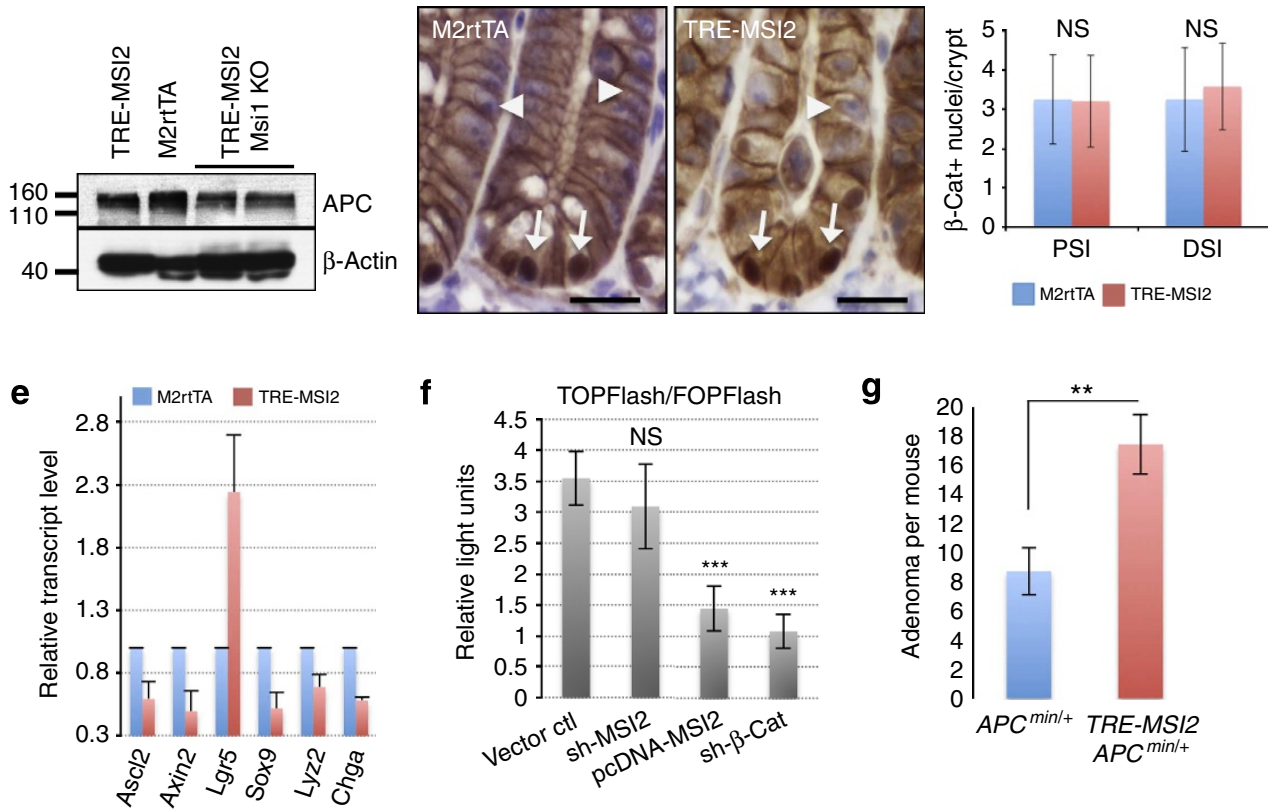

Figure 5 | MSI2 activation imposes an APC-loss gene signature in the absence of Wnt pathway activation. (a) Hierarchical clustering of transcriptome profiles from the intestinal epithelium of three control (M2rtTA) and three TRE-MS/2 mice $24 \mathrm{~h}$ after $2 \mathrm{mg} \mathrm{ml}^{-1}$ Dox induction. (b) GSEA of the transcriptome profiles showing a highly significant enrichment of APC loss gene signatures in TRE-MSI2 mice. Top panel: genes activated by both APC loss and MSI2 induction; bottom panel: genes suppressed on APC loss and MSI2 induction. FDR: false discover rate. (c) Western blot for APC in lysates of the intestinal epithelium of control (M2rtTA), TRE-MSI2 and TRE-MSI2-MsiTKO mice $48 \mathrm{~h}$ after $2 \mathrm{mg} \mathrm{ml}^{-1}$ Dox induction. (d) Immunohistochemical staining for $\beta$-catenin in control (M2rtTA) and TRE-MSI2 intestinal crypts $48 \mathrm{~h}$ after $2 \mathrm{mg} \mathrm{ml}^{-1}$ dox induction, with number of $\beta$-catenin ${ }^{+}$nuclei per crypt section quantified at right (PSI/DSI: proximal and distal small intestine). Error bars indicate the s.d. derived from at least 50 crypts from each mouse $(n=3$ mice per group, Student's t-test). (e) QRT-PCR analysis of Wnt target gene and differentiation-related gene expression in purified crypts from control (M2rtTA) and TRE-MSI2 intestinal crypts $48 \mathrm{~h}$ after $2 \mathrm{mg} \mathrm{ml}^{-1}$ dox induction. Error bars indicate the s.d., $n=3$. (f) $\beta$-Catenin/TCF transcriptional reporter activity (TOPFLash) normalized to activity of the reporter with a mutated binding element (FOPFlash) in HEK293 cells upon MSI2 knockdown (sh-MSI2), overexpression (pcDNA-MSI2) or $\beta$-catenin knockdown (sh- $\beta$-CAT) as a control. Error bars indicate the s.d. $(n=4)$. ${ }^{\star \star \star} P<0.0005$, Student's $t$-test. $(\mathbf{g})$ Frequency of adenomas in Apc $\mathrm{min} /+(n=4)$ and TRE-MSI2::Apc ${ }^{\mathrm{min} /+}(n=4)$ mice treated with low dose Dox $\left(0.1 \mathrm{mg} \mathrm{ml}^{-1}\right)$ in drinking water for 3 weeks. Error bars denote the s.d. ${ }^{\star \star} P<0.005$, Student's $t$-test.

(refs 29,30), and consistent with what we previously observed for MSI2 in haematopoietic cancers ${ }^{6}$ (Fig. 6a). The vast majority of transcripts bound by Msi2 in wild-type crypts were also bound by ectopic MSI2 (4,387/4,824, or 90\%; Fig. 6b). Conservation analysis of Msi2/MSI2-binding sites revealed that sites in coding sequences and UTRs were significantly conserved relative to flanking sequences; however, this was not true of intronic binding events, suggesting that Msi2/MSI2 binding of introns may represent promiscuous events (Fig. $6 \mathrm{c}$ and Supplementary Fig. 5a). Limiting analysis specifically to $3^{\prime}$ UTR-bound transcripts reveals a similar overlap between Msi2 targets in wild-type crypts and MSI2-bound targets in the
TRE-MSI2 epithelium (91\% of Msi2 targets also bound by ectopic MSI2, and $76 \%$ of TRE-MSI2 targets also bound by Msi2 in wild-type crypts; Supplementary Fig. 5b).

Msi2/MSI2 binding was, overall, not selective for the most highly expressed transcripts, and binding did not globally affect overall mRNA levels, as transcripts newly bound on MSI2 induction in the TRE-MSI2 epithelium exhibited no change in mRNA levels (Supplementary Fig. $5 \mathrm{c}-\mathrm{e}$ ). This is consistent with MSI2 RNA interaction promoting neither mRNA degradation nor stabilization. Binding motif analysis for Msi2 in wildtype (WT) crypts revealed that Msi2 bound a number of U-rich motifs, and the previously published Msil-binding motif 
a

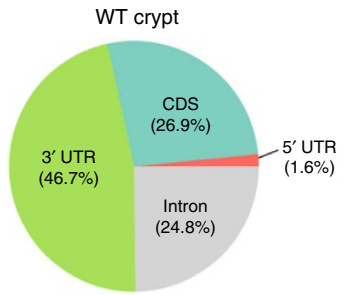

c

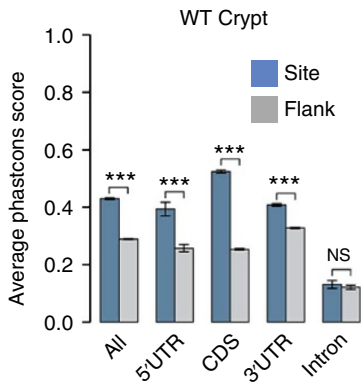

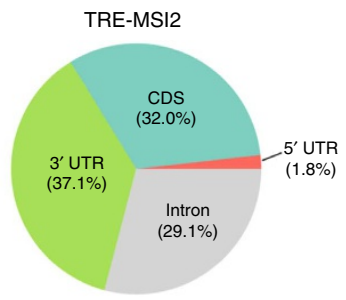

b

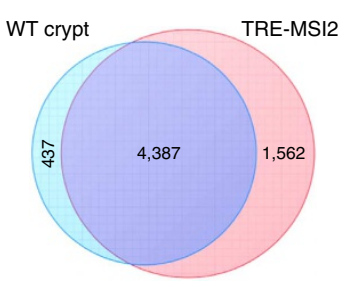

C

e

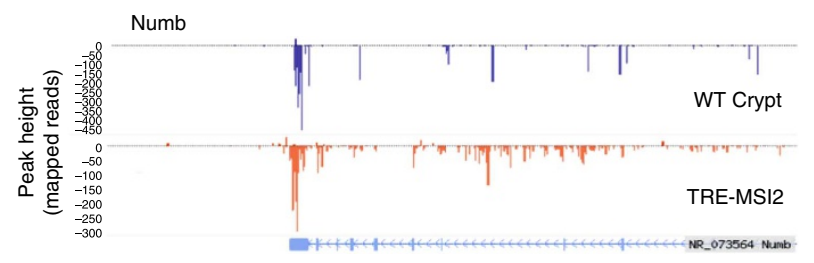

d

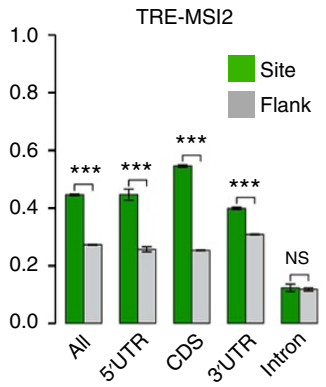

Matr

: : :UASAGUVUCCAA

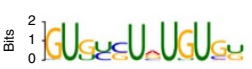

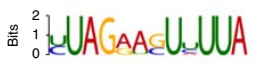

:

$\square$ 5'UTR $\square$ CDS $\square$ 5'UTR $\square$ Intron

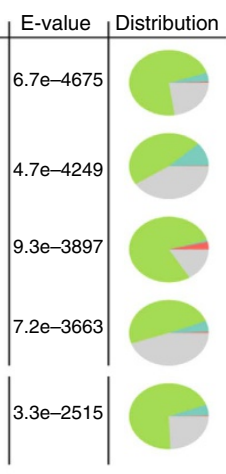

$\left|\begin{array}{l|l}\text { E-value } & \text { Distribution } \\ 6.7 \mathrm{e}-4675 \\ 4.7 \mathrm{e}-4249 \\ 9.3 \mathrm{e}-3897 \\ 7.2 \mathrm{e}-3663 \\ \mid \\ 3.3 \mathrm{e}-2515\end{array}\right|$

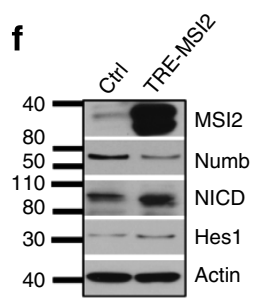

g

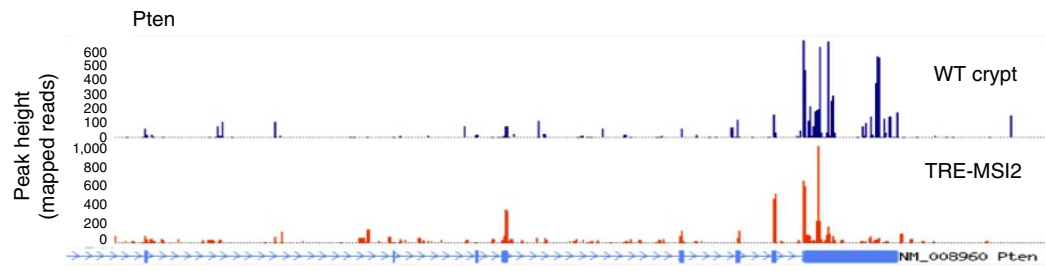

Figure 6 | Transcriptome-wide MSI2 RNA-binding analysis. (a) CLIP-Seq shows the distribution of Msi2/MSI2 RNA-binding events in wild-type intestinal crypts and the TRE-MSI2 epithelium. (b) Venn diagrams show overlap between Msi2/MSI2-bound transcripts in wild-type crypts (left) and the TRE-MSI2 epithelium (right) $24 \mathrm{~h}$ after $2 \mathrm{mg} \mathrm{ml}^{-1}$ Dox induction. (c) PhastCons conservation analysis of Msi2/MSI2 binding quantifying evolutionary conservation of binding sites relative to flanking sequences in all categories with the exception of intronic binding sites (these were not conserved relative to flanking sequences). Error bars represent $95 \%$ confidence intervals. ${ }^{\star \star \star} P<0.0005$, two-sample Kolmogorov-Smirnov test. (d) Msi2-binding motif identification and distribution in WT crypts. The previously identified Msi1-binding motif is the final motif pictured. (e) CLIP-Seq track showing Msi2/MSI2 binding to the $3^{\prime}$ UTR of the mRNA encoding Numb. (f) Western blot analysis of Numb, activated Notch (Notch intracellular domain, NICD) and the downstream Notch target gene Hes1 in control (M2rtTA), and the TRE-MSI2 intestinal epithelium. (g) CLIP-Seq track showing Msi2/MSI2 binding to the $3^{\prime}$ UTR of the mRNA-encoding Pten.

[(G/A)U(n)AGU ( $n=1-3)]$ (ref. 29), while not among the most significant, was still highly enriched and significant in the data (Fig. 6d). Electrophoretic mobility shift assays confirmed the interaction of recombinant human MSI2 with the dominant $3^{\prime}$ UTR motif 5'-ACCUUUUUAGAA-3' (Supplementary Fig. 5f). Global analysis of Msi2 RNA-binding targets revealed an enrichment in transcripts encoding proteins involved in protein localization and transport, ribosome biology and nucleotide/ ribonucleotide binding, consistent with what was observed previously for MSI2 in haematopoietic cancers ${ }^{6}$ (Supplementary Fig. 6a). Interestingly, mRNA targets involved in neurodegenerative diseases including Parkinson's, Alzheimer's and Huntington's were also significantly enriched, possibly reflecting a role for MSI2 in these disorders (Supplementary Fig. 6a).
We next examined the binding of Msi2 to transcripts that have previously been identified as Msil-binding targets in vitro, including Cdkn1a (encoding p21) and Numb ${ }^{29,30}$. Msi2/MSI2 bound both Cdkn1a and Numb mRNAs in their $3^{\prime}$ UTRs (Fig. 6e and Supplementary Fig. 6b). Interestingly, MSI2 induction had only modest effects reducing the Numb protein and activating Notch signalling, and no enrichment for a Notch expression signature was observed in our transcriptome profiles, indicating that Notch activation is not a major consequence of MSI2 induction.

These data validate our CLIP-Seq approach and indicate that Msi2 has an RNA-binding activity similar to the second mammalian Musashi family member, Msil. Given the observed binding of MSI2 to the transcripts encoding the tumour 
suppressors p21 and Numb, we examined the CLIP data set for other interactions between MSI2 and tumour suppressors. Remarkably, MSI2 bound several well-established intestinal tumour suppressions including Lrig1 (ref. 31), Bmpr1a (ref. 32) and Pten ${ }^{33,34}$ (Fig. 6f and Supplementary Fig. 6b). Interestingly, PTEN protein is lost in many CRCs; however, the PTEN gene is rarely the target of genetic inactivation ${ }^{35}$. Thus, it is tempting to speculate that binding of PTEN mRNA by MSI2 may account for the loss of the PTEN protein in PTEN-wild-type cancers.

MSI2 inhibits Pten and activates the mTORC1 pathway. PTEN is a well-known tumour suppressor in CRC and has been implicated in the regulation of $\mathrm{ISCs}^{33}$. Deletion of Pten in the context of APC deficiency accelerates adenoma formation and activates the PDK-AKT-mTORC1 pathway ${ }^{33,34,36}$. To investigate the possibility that MSI2-mediated transformation of the intestinal epithelium is a result of Pten inhibition, we examined Pten protein levels and observed a reduction on TRE-MSI2 induction (Fig. 7b). Pten is a phosphatase that dephosphorylates phosphatidylinositol (3,4,5)-triphosphate (PIP3), converting it to PIP2. Loss of Pten thus blocks the conversion of PIP3 to PIP2, leading to an accumulation of PIP3. Analysis of PIP3 in both total intestinal epithelial cells and specifically in Lgr5 ${ }^{+}$CBC stem cells reveals an increase in PIP3positive cells after TRE-MSI2 induction, consistent with an inhibition of Pten activity (Fig. 7b).

Accumulating PIP3 binds to PDK1, in turn activating the AKT-mTorc1 pathway ${ }^{37}$. Consistent with Pten suppression and mTORC1 activation downstream of MSI2 induction, GSEA revealed an inverse correlation between the TRE-MSI2 gene signature and the Rapamycin gene signature (Fig. 7c). Rapamycin is a potent inhibitor of the mTORC1 complex, and thus these findings indicate that the $\mathrm{mTORC} 1$ complex is being activated by MSI2 induction. We further examined activity of the pathway downstream of Pten and observed increased phosphorylation of AKT (Phospo-AKT ${ }^{(\mathrm{T} 308)}$ and Phospo-AKT ${ }^{(\mathrm{S} 43)}$, Fig. 7d), as well as increased mTORC1 activity, assessed by phosphorylation of S6 by 56 kinase and phosphorylation of 4EBP1 (Fig. $7 \mathrm{~d}$ and Supplementary Fig. 7a).

Ultimately, we asked whether MSI2-mediated mTORC1 activation functionally contributes to intestinal transformation in TRE-MSI2 mice. Since it is well established that mTORC1 activity is dispensable for intestinal homeostasis ${ }^{38,39}$, we blocked mTORC1 signalling in TRE-MSI2 mice with Rapamycin for 2 days before Dox induction of MSI2 for $48 \mathrm{~h}$. Phosphorylation of S6 was markedly reduced in Rapamycin-treated TRE-MSI2 mice (Supplementary Fig. 7b), confirming that mTORC1 activity was effectively inhibited. Strikingly, we found that the expanded proliferative zone within the crypts marked by Ki67 in TRE-MSI2 mice was fully rescued by the Rapamycin treatment (Fig. 7e,f). Furthermore, we observed that the increase in crypt density/crypt fission was also rescued in the Rapamycin-treated TRE-MSI2 mice, demonstrating that activation of mTORC1 functionally contributes the increased crypt fission and hyperplasia observed in TRE-MSI2 mice (Fig. 7g). In summary, these findings demonstrate that MSI2 is a potent oncogene capable of recapitulating phenotypes resulting from APC loss through the activation of mTORC1 in an unappreciated, Wnt-independent oncogenic pathway.

\section{Discussion}

Here we identify MSI2 as a potent oncogene in CRC. The current dogma of CRC initiation suggests that loss of the APC tumour suppressor results in the failure to degrade $\beta$-catenin, consequently resulting in constitutive activation of the Wnt signalling pathway. This process is believed to result in tumorigenesis only when it occurs in ISCs, as genetic ablation of APC in other cell types of the intestine does not initiate tumorigenesis ${ }^{13}$. In the current study we observe high levels of MSI2 in primary human CRCs, along with Msi2 induction upon the loss of APC in mouse models. Further, we observe that loss of MSI2 abrogates growth of CRC cells (both APC-wild-type and APC-negative), where its loss is as effective as loss of $\beta$-catenin in inhibiting CRC cell growth. These findings, coupled with our previous findings in acute and chronic myelogenous leukaemias ${ }^{4}$, indicate that MSI2 is upregulated in a number of cancers (possibly through a variety of mechanisms, as elevated MSI2 levels are found in cancers with distinct underlying genetic mutations), and that these cancers become dependent on oncogenic MSI2 activity.

In the current study we sought to model the consequences of MSI2 induction on the intestinal epithelium. Using a single-copy, drug-inducible MSI2 transgenic mouse model we are able to rapidly and robustly induce MSI2 activity throughout the intestinal epithelium. Strikingly, MSI2 activity alone was sufficient to phenocopy many aspects of APC loss in the intestinal epithelium (the exception being Paneth cell mislocalization), resulting in a dramatic expansion of the cryptproliferative zone, a block in differentiation and an increase in crypt fission ${ }^{18}$. This phenotype was also reflected at the molecular level, with unbiased GSEA of the MSI2-expressing epithelium identifying the APC loss gene signature among the most significantly enriched.

Given the dogma that APC loss drives intestinal cancer through constitutive Wnt pathway activation, we examined whether Wnt hyperactivation could contribute to the TRE-MSI2 phenotype and, surprisingly, found no evidence of Wnt pathway activation, either at the level of APC loss, nuclear $\beta$-catenin localization or $\beta$-catenin target gene activity. This result prompts a reassessment of the dogma that constitutive $\beta$-catenin activity is the sole oncogenic consequence of APC loss, a notion that is supported by recent evidence that constitutive $\beta$-catenin activation is not sufficient to recapitulate many phenotypes associated with APC loss ${ }^{28,40}$. Taken together, our findings suggest that MSI2 activation represents an oncogenic pathway downstream of APC loss and parallel to $\beta$-catenin activation.

This concept runs contrary to recent in vitro reports that the second mammalian MSI family member, MSI1, activates canonical Wnt signalling $24,41,42$, possibly reflecting differences between the in vivo setting used in the current study and the in vitro approaches taken in published studies, as well as tissuespecific differences, as only one of these studies examined CRC cells in vitro ${ }^{41}$. Given the evidence for functional redundancy between the two mammalian MSI proteins ${ }^{26}$, and that MSI1 appears to have an oncogenic role in CRC cell lines ${ }^{23}$, we also examined Msil expression in our TRE-MSI2 mouse model and observed a striking induction of Msil at the mRNA level; however we conclude, through Msil loss-of-function studies, that the oncogenic properties of MSI2 are not dependent on Msi1, although this certainly does not rule out an oncogenic role for Msi1/MSI1 independently of Msi2/MSI2.

The co-expression of both Msi proteins in stem cells of the intestinal crypt (this study and ref. 10), the lack of any major intestinal phenotype described in either germline Msi1 knockout mice $^{26}$ and our conditional Msi $1^{\text {flox/flox }:: V i l l i n C r e E R}$ mice (this study), along with the aforementioned functional redundancy of Msi proteins ${ }^{26}$ raises the intriguing possibility that Msi proteins act redundantly to maintain stem cell self-renewal in the ISC compartment, as we have previously observed for Msi2 in $\mathrm{HSCs}^{6}$. It will be important to address this possibility in future studies utilizing dual-Msi conditional knockout mice. 
a

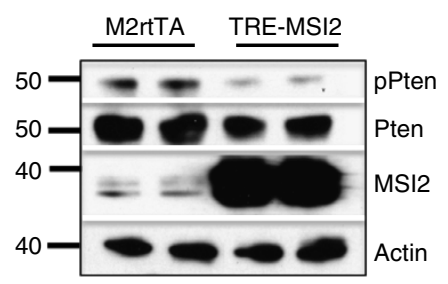

C

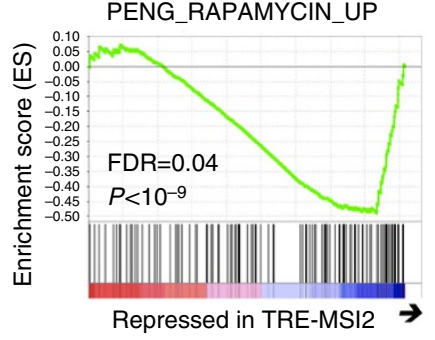

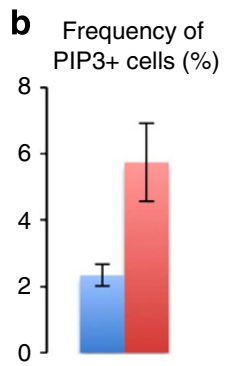

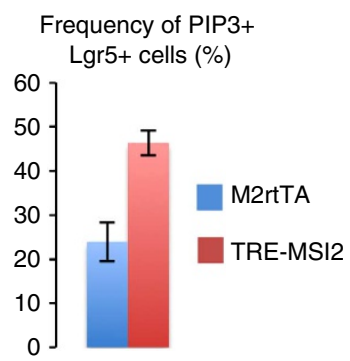

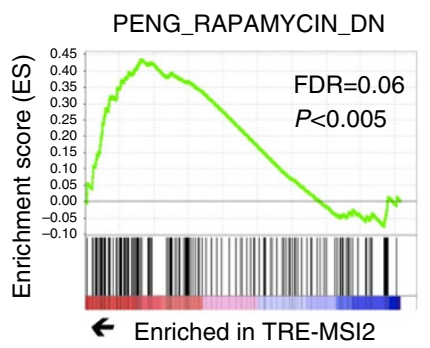

d
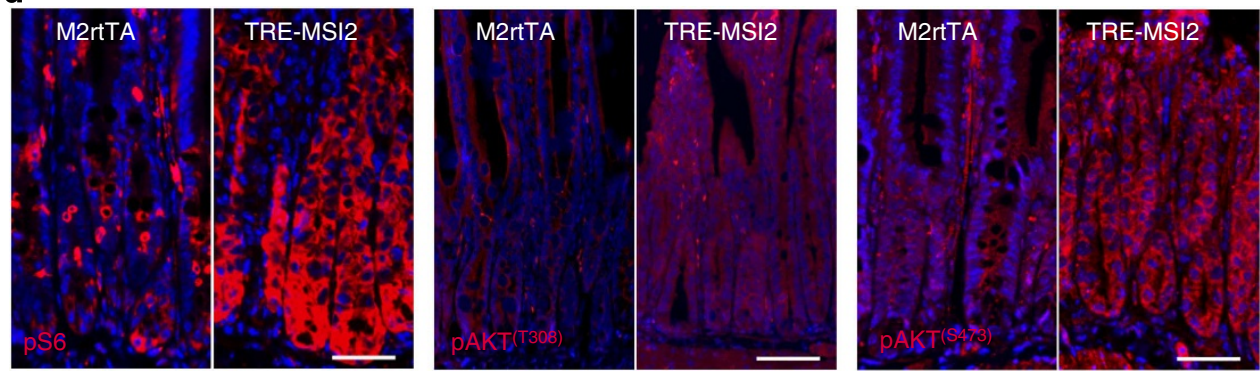

e

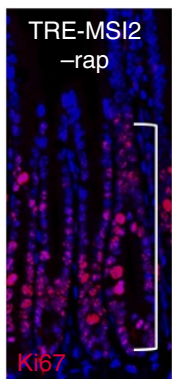

f

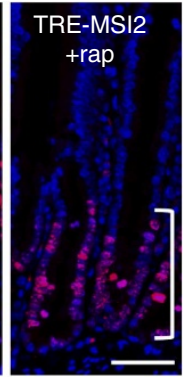

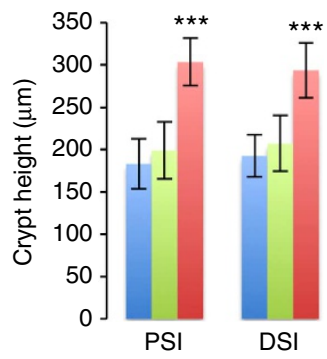

g

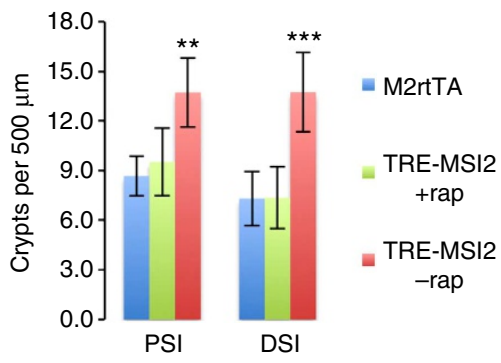

Figure 7 | Activation of the Pten-AKT-mTORC1 axis is necessary for MSI2 transformation of the intestinal epithelium. (a) Western blotting showing reduction of the Pten protein in TRE-MSI2 crypts versus controls (M2rtTA) $48 \mathrm{~h}$ after $2 \mathrm{mg} \mathrm{ml}^{-1}$ Dox induction. (b) Flow cytometric quantification of PIP3 ${ }^{+}$cell frequency in total intestinal epithelial cells (left) and Lgr5-eGFP + crypt base columnar stem cells in (right) between control (M2rtTA) and TRE-MSI2 mice $48 \mathrm{~h}$ after Dox induction ( $n=3$ mice per group), error bars indicate the s.d. (c) GSEA demonstrating an inverse correlation between the TRE-MSI2 transcriptome and Rapamycin-regulated genes. Genes upregulated by the Rapamycin treatment are suppressed by MSI2 (left), and genes suppressed by the Rapamycin treatment are upregulated by MSI2 induction. (d) Immunofluorescence for pS6, p-AKT (T308) and p-AKT (s473) showing activation of the AKT/mTORC1 signalling axis in TRE-MSI2 mice (scale bar, $50 \mu \mathrm{M}$ ). (e) Immunofluorescence staining for Ki67 in Dox-induced TRE-MSI2 mice treated with or without Rapamycin (scale bar, $50 \mu \mathrm{M}$ ). (f,g) Qualification of crypt height in $\mathbf{f}$ and crypt frequency in $\mathbf{g}$ in control (M2rtTA), TRE-MSI2 and Rapamycin-treated TRE-MSI2 mice. Error bars denote s.d. Crypt height was measured based on 40 crypts in each of three independent pairs of mice. Crypt frequency was scored in 20 randomly selected areas in proximal and distal intestine (PSI and DSI, respectively) in each of three independent pairs of mice. ${ }^{\star \star} P<0.005,{ }^{\star \star \star} P<0.0005$.

Transcriptome-wide RNA-binding analysis of endogenous Msi2 in intestinal crypts, ectopic MSI2 in the intestinal epithelium and of MSI2 in human leukaemic cell lines offers insight into the tissue-specific versus common functions of MSI2 in these tissues. In the intestinal transcriptome analysed in this study and the leukaemic transcriptome analysed in ref. 6, MSI2 bound to transcripts involved in RNA biogenesis and metabolism, suggesting that MSI regulation of these fundamental biological processes may be conserved across tissues. Pathway analysis in haematopoietic tissues indicates that MSI2 primarily acts to modulate TGF- $\beta$ signalling, whereas in the intestinal epithelium, the Pten-AKT-mTORC1 axis was identified as being a major target of MSI2 activity, indicating that MSI2 may have cell-typespecific functions (although the mTOR pathway was also 
identified as an MSI2 target in the haematopoietic system, it appears not to be as dramatically affected as in the intestinal epithelium).

Conceptually, this phenomenon is not unexpected as RNAbinding protein activity must, by definition, be restricted to the repertoire of transcripts expressed in a particular cell type. This is in contrast to transcription factors, whose DNA targets are identical in all cell types, epigenetic-regulated accessibility and cofactor binding notwithstanding. Thus, MSI2 and other RNAbinding proteins might serve as hubs of signal integration by acting on mRNAs in the cytoplasm, with the possibility of celltype-specific function depending on the mRNA repertoire available for binding in any given cell type. In the current study, we identify the Pten-AKT-mTORC1 axis as a major MSI2 target with functional relevance to the observed phenotype; however, we note that MSI2 binds numerous additional tumour suppressors with known roles in CRC, including Cdknla, Lrig1, Bmprla and Numb. At least two of these targets (Bmprla and Lrig1, in addition to Pten) are also known repressors of $m$ Torc1, and thus the effects of MSI2 on mTorcl activity may be the result of pleiotropic inhibition of these tumour suppressors. While such pleiotropic inactivation of tumour suppressors may make MSI2 a potent oncogene, it also represents an attractive point for therapeutic intervention. In principle, MSI2 inhibition may allow for the concerted re-activation of this cadre of colorectal tumour suppressors. Thus, the viability of MSI2 as a drug target will be of great interest moving forward in the fight against CRC, and likely a variety of other cancers.

\section{Methods}

Mouse strains. All procedures involving mice were reviewed and approved by the Institutional Animal Care and Use Committee of the University of Pennsylvania (Animal Welfare Assurance Reference Number no. A3079-01, approved protocol no. 803415 granted to Dr Lengner) and were in accordance with the guidelines set forth in the Guide for the Care and Use of Laboratory Animals of the National Research Council of the National Institutes of Health.

The generation of TRE-MSI2 mice is described in ref. 4. Briefly, the human MSI2 cDNA (open biosystems clone IDno. 5942 Accession CV027635) under control of the tetracycline operator with a minimal CMV promoter was targeted to safe-haven chromatin downstream of the Collagen1a1 locus in $\mathrm{KH} 2$ embryonic stem cells also harbouring the modified reverse tetracycline transactivator (M2rtTA) targeted to and under transcriptional control of the ROSA26 locus ${ }^{43}$. Lgr5-eGFP-CreER mice were obtained from Jackson Laboratories (stock no. 008875). Conditional loss-of-function $M s 1^{\text {flox/flox }}$ mice will be described in detail elsewhere. Briefly, the two exons, including the transcriptional start site of Msi1, were targeted with flanking LoxP sites resulting in the 2lox, or floxed locus.

Deletion of this region results in the loss of the transcriptional start site and a frameshift in the Msil-coding sequence. Proper recombination at both the $5^{\prime}$ and $3^{\prime}$ ends of the arms of homology was confirmed by Southern blotting. Mice generated from 2lox ES cells were bred to homozygosity and crossed with Villin-CreER mice ${ }^{17}$. Msil was conditionally deleted by administration of five consecutive doses of Tamoxifen $\left(50 \mathrm{mg} \mathrm{kg}^{-1}\right)$ in corn oil (Sigma), and loss of Msi1/Msil was confirmed by Southern blotting, quantitative reverse transcription-PCR (qRTPCR) and immunostaining. Dox induction of MSI2 in TRE-MSI2::Msi ${ }^{\text {flox/flox }::}$ VillinCreER mice was initiated 5 days after the final dose of Tamoxifen.

TRE-B-CatS33 (ref. 28) mice were a kind gift of Dr Konrad Hochedlinger.

For ablation of $A P C, A P C^{\text {flox/flox }}$ mice ${ }^{44}$ were crossed with Villin-CreER mice and $A P C$ was deleted with four consecutive daily doses of Tamoxifen $\left(50 \mathrm{mg} \mathrm{kg}^{-1}\right)$ in corn oil (Sigma). Epithelial RNA was isolated 5 days after the initial Tamoxifen dose and processed for qRT-PCR as described below.

The generation of Msi2 flox/flox mice is described in ref. 6. Gamma irradiation after Msi2 deletion in Msi flox/flox::Villin-CreER mice was performed on at least four mice in each of knockout and control groups, treated with five consecutive doses of Tamoxifen $\left(50 \mathrm{mg} \mathrm{kg}^{-1}\right)$ in corn oil (Sigma) followed by 5 days of recovery and exposure to $12 \mathrm{~Gy}$ whole-body $\gamma$-irradiation. Controls and experimental mice were sex-matched littermates. Intestines were washed with PBS, fixed in $4 \%$ paraformaldehyde, paraffin-embedded and sectioned. Surviving crypts were defined as 10 or more adjacent chromophilic cells and a lumen in haematoxylin and eosin-stained sections from the intestinal epithelium.

Dox induction and isolation of the intestinal epithelium. For Dox induction experiments of control $\left(M 2 r t T A^{+/+}\right)$and TRE-MSI2 $2^{+/-}:: M 2 r t T A^{+1+}$ mice, $2 \mathrm{mg} \mathrm{ml}^{-1}$ Dox (Doxycycline hyclate, Sigma) was added to the drinking water

along with $1 \% \mathrm{w} / \mathrm{v}$ sucrose of mice $8-10$ weeks of age. All experiments except tumour formation in TRE-MSI2::APC ${ }^{\mathrm{min} /+}$ mice utilized the $2-\mathrm{mg} \mathrm{ml}^{-1}$ Dox dosage in mice homozygous for M2rtTA and heterozygous for the TRE-MSI2 allele. Most experiments were conducted after $48 \mathrm{~h}$ of exposure to Dox-containing drinking water, with the exception of transcriptome profiling, which was carried out $24 \mathrm{~h}$ after the initiation of Dox treatment. For $A P C^{\mathrm{min} /+}$ experiments, TRE-MSI2 $2^{+1-}:: A P C^{\mathrm{min} /+}:: M 2 r t T A^{+/-}$or APC $\mathrm{min} /+:: M 2 r t T A^{+/-}$(in these experiments the M2rtTA was heterozygous to reduce MSI2 dosage) were administered $0.1 \mathrm{mg} \mathrm{ml}^{-1}$ of Dox in the drinking water for 3 weeks starting at 2 months of age while being fed low-protein/high-fat diet (Research Diets, D12079B). For isolation of whole-intestinal epithelial cells, the mouse intestine was cut longitudinally and washed two to three times with ice-cold Dulbecco's phosphate buffered saline (DPBS), then cut into small pieces (3-7 mm long) and incubated for $1 \mathrm{~h}$ at $4^{\circ} \mathrm{C}$ in DPBS containing $2 \mathrm{mM}$ EDTA and $0.2 \mathrm{mM}$ dithiothreitol on a rotating platform, and collecting suspended cells after gentle vortexing. For isolation of intestinal crypts, mouse small intestine was washed with DPBS as above. The villi were scraped using a hemocytometer coverslip. The crypts were released by incubation for $30 \mathrm{~min}$ at $4{ }^{\circ} \mathrm{C}$ in DPBS containing $2 \mathrm{mM}$ EDTA and gentle vortexing. Isolated crypts were counted and pelleted as described in ref. 45 .

Flow cytometry. The intestine was cut open longitudinally and incubated with $5 \mathrm{mM}$ EDTA-HBSS solution at $4{ }^{\circ} \mathrm{C}$ for $30 \mathrm{~min}$. Single cell suspension was generated with Dispase (BD Biosciences, San Jose, CA). Flow cytometry analysis was performed with BD LSR Fortessa cell analyzer (BD Biosciences). $4^{\prime}$,6-diamidino-2-phenylindole (DAPI)-negative cells were selected, and then gated for single cell based on Forward-scatter height versus forward-scatter width and side-scatter height versus side-scatter width profiles. The size of the nozzle for all sorting is $100 \mu \mathrm{m}$ ( $20 \mathrm{psi})$. Lgr5 + stem cells were quantified by flow cytometry in TRE-MSI2::Lgr5-eGFP-IRES-CreER and M2rtTA::Lgr5-eGFP-IRES-CreER control mice after $48 \mathrm{~h}$ of the Dox treatment.

EdU labelling and flow cytometry. TRE-MSI2::Lgr5-eGFP-IRES-CreER and M2rtTA::Lgr5-eGFP-IRES-CreER control mice were treated with $2 \mathrm{mg} \mathrm{ml}^{-1}$ Dox (Sigma) and were injected with $0.15 \mathrm{mg}$ of 5 -EdU (Life Technologies) 2 or $12 \mathrm{~h}$ before killing and intestinal isolation. The intestine was cut open longitudinally and washed twice with ice-cold PBS. The intestine was incubated with $30 \mathrm{mM}$ EDTA (EDTA, Sigma) and $1.5 \mathrm{mM}$ DTT (Sigma) in HBSS at $4{ }^{\circ} \mathrm{C}$ for $20 \mathrm{~min}$. Then, it was moved to $30 \mathrm{mM}$ EDTA in $\mathrm{HBSS}$ at $37^{\circ} \mathrm{C}$ for $10 \mathrm{~min}$ and dissociated by pipetting vigorously. Single-cell suspension was generated with $0.8 \mathrm{mg} \mathrm{ml}^{-1}$ Dispase $(\mathrm{GIBCO})^{46}$. Cells were fixed with Click-iT fixative (Life Technologies) for $15 \mathrm{~min}$ at room temperature and stained with FITC-anti-GFP (fluorescein isothiocyanateanti-green fluorescent protein; Abcam) for $30 \mathrm{~min}$ on ice and washed twice with Click-iT permeabilization and wash reagent. For Click-it labelling, cells were incubated with $\mathrm{CuSO}_{4}$ and Alexa fluor 647 azide dye as per the user manual and cells were analysed using flow cytometry. Flow cytometry was performed on an LSR Fortessa, and the Flowjo software was used for data analysis.

PIP3 flow cytometry. Intestinal epithelial cells were suspended in PBS containing $1 \mu \mathrm{ml}^{-1}$ of fixable viability dye eFluor 450 (Affymetrix ebioscience). Cells were incubated on ice for $30 \mathrm{~min}$ and were washed two times with PBS. Then, cells were fixed and permeabilized using Cytofix/Cytoperm solution (BD PharMingen), stained with FITC-anti-GFP (Abcam) and biotinylated anti-PIP3 (Echelon Inc.) for $30 \mathrm{~min}$ on ice and washed twice. They were incubated with StreptavidinAPC(Biolegend) for $30 \mathrm{~min}$ on ice and washed twice. Flow cytometry was performed on an LSR Fortessa, and the Flowjo software was used for data analysis.

Administration of Rapamycin. Rapamycin (LC Laboratories) was administered by daily intraperitoneal injection ( $4 \mathrm{mg}$ per $\mathrm{kg}$ of body weight) for 5 days. It was reconstituted in absolute ethanol at $10 \mathrm{mg} \mathrm{ml}^{-1}$ and diluted in $5 \%$ Tween-80 (Sigma) and 5\% PEG-400 (Hampton Research) before injection. The final volume of all injections was $200 \mu \mathrm{l}$. Dox was administered to TRE-MSI2 mice as above for $48 \mathrm{~h}$ after the third dose of Rapamycin.

\section{Crypt organoid culture and qRT-PCR from the intestinal epithelium and}

organoids. Crypt culture was performed as previously described in ref. 45. After intestinal crypt isolation, a total of 500 crypts were mixed with $50 \mu$ of Matrigel (BD Bioscience) and plated in 24-well plates. After polymerization of Matrigel, $500 \mu \mathrm{l}$ of crypt culture medium (Advanced DMEM/F12 containing $50 \mathrm{ng} \mathrm{ml}^{-1}$ EGF (Invitrogen), $1 \mu \mathrm{g} \mathrm{ml}^{-1} \mathrm{R}$-spondin (Wistar Institute protein production facility), $100 \mathrm{ng} \mathrm{ml}^{-1}$ Noggin (Peprotech) and $3 \mu \mathrm{M}$ GSK-3 inhibitor CHIR99021 (Stemgent) was added. After 2 -day culture at $37-{ }^{\circ} \mathrm{C}$ incubator, the organoids were treated with $2 \mu \mathrm{g} \mathrm{ml}^{-1}$ Dox and harvested for qRT-PCR analysis $72 \mathrm{~h}$ later.

RNA was isolated from organoids, crypts or total epithelium, using QIAGEN RNeasy mini plus kits. Total RNA $(1 \mu \mathrm{g})$ was used for cDNA synthesis. Primers used in $\mathrm{qRT}-\mathrm{PCR}$ are as follows:

Forward $\left(5^{\prime}-3^{\prime}\right)$

Ascl2 ATCTGCCCGGAGCATGGAA

Axin2 GATCCACGGAAACAGCTGAA
Reverse $\left(5^{\prime}-3^{\prime}\right)$ CACTGCTGCAGGACTCCCTA AGCCGGAACCTACGTGATAA 
Chga CCCGAAGTGACTTTGAGGAA

Gapdh AGACGGCCGCATCTTCTT

Lgr5 ACGCCTTTGGAAACCTCTCC

Lyz2 GTGACAGTCAGCCAACACAA

Msil GCCATGCTGATGTTCGACAA

Msi2 GCGATGCTGATGTTCGACAA

Sox9 AGTACCCGCATCTGCACAA
ATGGCTGACAGGCTCTCTA TTCACACCGACCTTCACCAT CAGGCTGTGGAGTCCATCAAA TGGCTGAAGAACTGACCTACA CTACGATGTCCTCGCTCTCAA TCTCCACAACGTCTTCATTCTCA GTCTCTTCTCGCTCTCGTTCA

Histological analyses. Intestines were washed with DPBS, fixed in $10 \%$ formalin, paraffin-embedded and sectioned. Haematoxylin, eosin, Alcian blue and alkaline phosphatase staining were performed in the Morphology Core of the Penn Center for Molecular Studies in Digestive and Liver Diseases. For immunohistochemistry staining, antigen retrieval was performed by heating slides in $0.01 \mathrm{M}$ citrate buffer (pH 6) with a pressure cooker. The sections were then immunostained by the $A B C$ peroxidase method (Vector Laboratories) with diaminobenzidine as the enzyme substrate and haematoxylin as a counterstain. For detection of nuclear $\beta$-catenin, antibody clone 15B8 (Sigma; 1:1,000) was used in combination with the MOM kit (Vector Laboratories). For immunofluorescence staining, paraffin sections were pretreated in $0.01 \mathrm{M}$ citrate buffer $(\mathrm{pH} 6)$ with a pressure cooker, and incubated in primary antibodies, then incubated with Cy2- or Cy3-conjugated fluorescent secondary antibodies (Jackson Laboratory) and counterstained with DAPI in mounting media (Vector Laboratories). For amplification of signal from Msil and Msi2, we used a Biotin-Streptavidin system. The following antibodies were used: Ki67 (1:1,250, Leica), Msi1 (1:400, MBL), Msi2 (1:200, Novus Biologicals), Lysozyme (1:50, Santa Cruz), Chromogramin A (1:1,000, Abcam), GFP (1:200, Abcam), pS6 (1:200; Cell Signaling), p-AKT (T308; 1:200; Cell Signaling), p-AKT (S473; 1:200; Cell Signaling), p-4EBP1 (T37/46; 1:200; Cell Signaling).

CLIP-Seq. Clip libraries were made as previously described in ref. 47 with minor modification. Total intestinal epithelial cells from TRE-MSI2 mice treated with Dox for $24 \mathrm{~h}$ were isolated as above, and wild-type intestinal crypts were isolated as above. For crosslinking, cell suspension was exposed to two pulses of $265 \mathrm{~nm}$ UV light at $400 \mathrm{~mJ} \mathrm{~cm}^{-2}$ in a Stratalinker (Model 2400, Stratagene). Epithelial cells were then lysed using PXL buffer (PBS, 01\% SDS, $0.5 \%$ deoxycholate, $0.5 \%$ NP- 40 , plus protease inhibitor and RNAsin). The lysates were sequentially treated with DNaseI and RNase, and spin in ultramicrocentrifuge at 40,000 g for $20 \mathrm{~min}$. The supernatant was added to protein A Dynabeads (Dynal, 100.02) conjugated with MSI2 antibody (EMD Millipore 03-115) and incubated for $4 \mathrm{~h}$ at $4{ }^{\circ} \mathrm{C}$. The 32P- $\gamma-$ ATP-labelled $3^{\prime}$ RNA (RL-3) linker was ligated to the RNA fragment on beads overnight at $16^{\circ} \mathrm{C}$. The beads were re-suspended in $30 \mu$ of Novex loading buffer (without reducing agent), and separated with Novex NuPAGE 10\% Bis-Tris gel and transferred to S\&S BA-85 nitrocellulose membrane. After overnight exposure, an $\sim 50-\mathrm{KDa}$ band was visualized and the corresponding membrane was cut into small pieces. The RNA was released by proteinase $\mathrm{K}$ digestion and isolated using RNA phenol and $\mathrm{CHCl} 3$ solution. The $5^{\prime} \mathrm{RNA}$ (RL-5) linker was ligated into the RNA fragments. The RNA was transcribed into complementary DNA using RTPCR and amplified using Re-PCR with Solexa fusion primers. The CLIP library underwent single-end sequencing on an Illumina hiSeq2000 at the University of Pennsylvania Functional Genomics Core. RL-3: 5'-OHGUGUCAGUCACUUCC AGCGG-3' - puromycin; RL-5: 5'-OHAGGGAGGACGAUGCGG-3'-OH. Adapter sequences were removed from the $3^{\prime}$ end using cutadapt (http://journal.embnet. org/index.php/embnetjournal/article/view/200) with options '-a GTGTCAGTCAC TTCCAGCG -e $0.06-\mathrm{O} 6-\mathrm{m} \mathrm{12}$. Reads were then mapped to the mouse (mm9) transcriptome and genome using Tophat ${ }^{48}$ with options "--read-mismatches 1 --read-gap-length 1 --read-edit-dist 1 --max-multihits 100 --b2-very-sensitive --transcriptome-max-hits 100 --no-coverage-search --no-novel-juncs'. Peaks were called using Piranha ${ }^{49}$ with a window size of $200 \mathrm{bp}$. Motifs were identified using the MEME software suite ${ }^{50}$.

Transcriptome profiling. Total RNA was isolated from total mouse small intestinal epithelial cells from three M2rtTA and three TRE-MSI2 mice administered Dox for $24 \mathrm{~h}$ in the drinking water (as above) using TRIzol Reagent (Life Technologies) according to the manufacturer's instructions. Total RNA was DNAse-treated with an RNAse-free DNAse kit (Zymo Research). Purified RNA was submitted to the University of Pennsylvania Molecular Profiling Core, where samples were labelled and hybridized to Affymetrix Mouse Gene 1.0ST arrays. Microarray data were analysed using the Partek Genomics SuiteTM software. Following RMA background subtraction and normalization, a one-way analysis of variance (ANOVA) between controls (M2rtTA) and MSI2-induced (TRE-MSI2) was run to compute $P$ values of significance and F-statistic for each probe set. $q$ value, a measure of FDR, was computed within the Significance Analysis of Microarrays software for each probe set by running an unpaired $t$-test. The FDR values were integrated with the one-way ANOVA results. Genes that were significant at FDR cutoff of 5\% and changed at least twofold in either direction in the MSI2 induction group when compared with the control group were selected as the set of differentially expressed genes. This set of 836 unique genes and 6 samples were subjected to agglomerative hierarchical clustering analysis. Log2 intensities were median-centred across samples. Euclidean distance was used as the dissimilarity metric and average linkage method as clustering strategy. Results were visualized as intensity heatmap (Fig. 5a).

For analysis of direct $\beta$-catenin target gene expression in Fig. $5 f$, genes with published evidence for direct regulation by $\beta$-catenin demonstrated by chromatin immunoprecipitation were selected. These genes include Edn1, Fra-1 (Fosl1), c-Myc, Met, c-Jun, Pinx1, Tcf4, Egfr, Klf5, Cyclin D (Ccnd1), Mycbp, Mmp7, Cdkn2a, Vegfa, SNAI1, Fgf18, Gja1 (connexin-43), Axin2, Claudin1, Runx2, Lef1, Bglap, ENPP2, Nrcam, DLK1, Vcan, Fgf4, Fn1, Tcf1, (Hnf1a), Lgr5, Ppard, Sp5, Ovol1, Id2, L1CAM, Cdh1 (E-cadherin), Fst, Tnfrsf19, Neurod1, Ctla4, Wisp1, Fzd7, Mitf, Pou3f2, Gbx2, Nkx2-2, Neurog1, Eda, T, Pitx2, Btrc, Cdx1, Birc5 (survivin), Pkd1, Pml, and Lect2 (chemotaxin2).

Oncomine and TCGA. Using Oncomine analysis, the MSI2 expression level was analysed in the Kaiser colon database. The database includes full transcriptome profiles of 105 samples: Control (5); caecum adenocarcinoma (17); colon adenocarcinoma (41); colon mucinous adenocarcinoma (13); colon signet ring cell adenocarcinoma (2); colon small cell carcinoma (2); rectal adenocarinoma (8); rectal mucinous adenocarcinoma (4); rectal signet ring cell adenocarcinoma (1); rectosigmoid adenocarcinoma (10); and rectosigmoid mucinous adenocarcinoma (2; ref. 51). For TCGA analysis, mRNA expression levels from RNA-Seq on TCGA COAD tumour samples (314 solid tissue samples from healthy individuals and 314 tumour samples) were analyzed. Expression values were obtained by trimmed mean of M-values (TIMM) normalization of read counts in genes. Fold changes for MSI2 in matched tumour/control RNA-Seq sample pairs from TCGA COAD were calculated (total of 26 patients.) Distribution of MSI2 fold changes in tumour/ control pairs for 26 individuals plotted are in red (intraindividual comparison). Distribution of MSI2 fold changes between control/control comparisons for 26 distinct individuals plotted in grey (interindividual comparison).

Cell proliferation assays. SW48 (ATCC stock number CCL-231) and HT29 (ATCC stock number HTB-38) were seeded in 96-well plates at a density of 1,500 and 2,000 cell and cultured in DMEM supplemented with $10 \%$ FBS, $1 \%$ sodium pyruvate, $1 \%$ penicillin/streptomycin and $1 \%$ L-glutamine $24 \mathrm{~h}$ before infection. For infection, the medium was removed, and cells were gently washed with PBS. Viral supernatant $(100 \mu \mathrm{l})$ containing pSICO-eGFP-shScramble, -sh-MSI2, -sh-BCAT and -sh-MSI2 \& -sh-BCAT. Cell proliferation assays were performed using MTT (3-[4, 5-dimethylthiazol-2-yl]-2, 5-diphenyltetrazolium bromide) assays. Absorbance was measured at $570 \mathrm{~nm}$. All data ware expressed as a mean from at least three independent biological experiments with $n=4$ per condition per experiment.

Xenograft assays. Six-week-old female nude mice were obtained from the Stem Cell and Xenograft Core at UPENN. Stably infected CRC cells were trypsinized and suspended in PBS. A total volume of $0.2 \mathrm{ml}$ containing $2-5 \times 10^{6}$ cells and $25 \%$ volume MatriGEL was injected subcutaneously into the mouse flank. Tumour size was measured using a Vernier caliper. Tumour volumes were calculated using the formula $V=1 / 2\left(L \times W^{2}\right)$, where $L$ is length (longest dimension) and $W$ is width (shortest dimension) of the tumour. Moribund animals were killed according to the protocols of the University of Pennsylvania. Tumour growth rates in the xenograft experiment were evaluated by fitting a linear mixed effects model on the $\log _{10}$-transformed tumour volume with days, experiment indicator (MSI short hairpin RNA (shRNA) versus control shRNA) and interaction between days and experiment included as independent variables.

TOPFlash assays. TOP/FOPFlash assays were carried out using the Dual-Luciferase Reporter Assay System (Promega), 293 cells were seeded in 24 -well plate $12 \mathrm{~h}$ before co-transfection with 1,200 ng scrambled hairpin, Sh-MSI2, Sh-BCAT or pcDNA-MSI2 and 400 ng M50 Super $8 \times$ TOPflash or M50 Super $8 \times$ FOPflash reporter plasmids per well (Addgene plasmids 12456 and 12457). FugeneHD was used to mediate co-transfection, and the media contained $3 \mathrm{nM}$ CHIR99021 GSK3 $\beta$ inhibitor to insure Wnt pathway activation. The Renilla luciferase reporter vector pRL-SV40 $(200 \mathrm{ng})$ was simultaneously transfected as the control for Firefly luciferase in TOP/FOP vectors. Cells were harvested $48 \mathrm{~h}$ after transfection. Experiments were performed at least twice with $n=4$ wells as technical replicates in each experiment.

Western blots. Cells were lysed in RIPA buffer with protease inhibitors (Roche). After quantification using a BCA protein assay kit (Pierce), $40 \mu \mathrm{g}$ of total protein was separated with $10 \%$ SDS-PAGE under denaturing conditions and were transferred to polyvinylidene difluoride membranes (GE Healthcare). The membranes were blocked in 5\% BSA (Sigma) and then incubated with a Msi2 primary antibody (1:1,000; Novus Biologicals), Msil (1:1,000; MBL), Notch intracellular domain (1:1,000; Abcam), Hes1 (1:1,000; Abcam), $\beta$-actin (1:10,000; Abcam), Pten (1:1,000; Cell Signaling) or APC (1:200; Santa Cruz sc-896) followed by incubation with a secondary antibody conjugated with horseradish peroxidase (HRP; 1:2,000 Cell Signaling) together with an HRP-conjugated primary antibody for $\beta$-actin (1:10,000; Sigma). Immunoreactive proteins were visualized using LumiGLO chemiluminescent substrate (Pierce). Raw scans of western blots in Figs $4 c, 5 c$, $6 \mathrm{f}$ and $7 \mathrm{a}$ can be found in the Supplementary Information accompanying the manuscript. 


\section{References}

1. Okano, H. et al. Function of RNA-binding protein Musashi-1 in stem cells. Exp. Cell Res. 306, 349-356 (2005).

2. Sakakibara, S. et al. Mouse-Musashi-1, a neural RNA-binding protein highly enriched in the mammalian CNS stem cell. Dev. Biol. 176, 230-242 (1996).

3. Sakakibara, S., Nakamura, Y., Satoh, H. \& Okano, H. Rna-binding protein Musashi2: developmentally regulated expression in neural precursor cells and subpopulations of neurons in mammalian CNS. J. Neurosci. 21, 8091-8107 (2001).

4. Kharas, M. G. et al. Musashi-2 regulates normal hematopoiesis and promotes aggressive myeloid leukemia. Nat. Med. 16, 903-908 (2010).

5. Ito, T. et al. Regulation of myeloid leukaemia by the cell-fate determinant Musashi. Nature 466, 765-768 (2010).

6. Park, S. M. et al. Musashi-2 controls cell fate, lineage bias, and TGF-beta signaling in HSCs. J. Exp. Med. 211, 71-87 (2014).

7. He, L. et al. Musashi2 predicts poor prognosis and invasion in hepatocellular carcinoma by driving epithelial-mesenchymal transition. J. Cell Mol. Med. 18, 49-58 (2014).

8. Li, L. et al. Expression of seven stem-cell-associated markers in human airway biopsy specimens obtained via fiberoptic bronchoscopy. J. Exp. Clin. Cancer Res. 32, 28 (2013).

9. Hope, K. J. et al. An RNAi screen identifies Msi2 and Prox1 as having opposite roles in the regulation of hematopoietic stem cell activity. Cell Stem Cell 7, 101-113 (2010).

10. Potten, C. S. et al. Identification of a putative intestinal stem cell and early lineage marker; musashi-1. Differentiation 71, 28-41 (2003).

11. Li, D. et al. Msi-1 is a predictor of survival and a novel therapeutic target in colon cancer. Ann. Surg. Oncol. 18, 2074-2083 (2011).

12. Fan, L. F. et al. Expression of putative stem cell genes Musashi-1 and betalintegrin in human colorectal adenomas and adenocarcinomas. Int. J. Colorectal Dis. 25, 17-23 (2010).

13. Barker, N. et al. Crypt stem cells as the cells-of-origin of intestinal cancer. Nature 457, 608-611 (2009).

14. Miyoshi, Y. et al. Somatic mutations of the APC gene in colorectal tumors: mutation cluster region in the APC gene. Hum. Mol. Genet. 1, 229-233 (1992).

15. Nagase, H. et al. Screening for germ-line mutations in familial adenomatous polyposis patients: 61 new patients and a summary of 150 unrelated patients. Hum. Mutat. 1, 467-473 (1992).

16. Kinzler, K. W. et al. Identification of FAP locus genes from chromosome 5q21. Science 253, 661-665 (1991).

17. el Marjou, F. et al. Tissue-specific and inducible Cre-mediated recombination in the gut epithelium. Genesis 39, 186-193 (2004).

18. Sansom, O. J. et al. Loss of Apc in vivo immediately perturbs Wnt signaling differentiation, and migration. Genes Dev. 18, 1385-1390 (2004).

19. van de Wetering, M. et al. The beta-catenin/TCF- 4 complex imposes a crypt progenitor phenotype on colorectal cancer cells. Cell 111, 241-250 (2002).

20. Barker, N. et al. Identification of stem cells in small intestine and colon by marker gene Lgr5. Nature 449, 1003-1007 (2007).

21. Wasan, H. S. et al. APC in the regulation of intestinal crypt fission. J. Pathol. 185, 246-255 (1998).

22. Asai, R., Okano, H. \& Yasugi, S. Correlation between Musashi-1 and c-hairy-1 expression and cell proliferation activity in the developing intestine and stomach of both chicken and mouse. Dev. Growth Differ. 47, 501-510 (2005).

23. Sureban, S. M. et al. Knockdown of RNA binding protein musashi-1 leads to tumor regression in vivo. Gastroenterology 134, 1448-1458 (2008).

24. Rezza, A. et al. The overexpression of the putative gut stem cell marker Musashi-1 induces tumorigenesis through Wnt and Notch activation. J. Cell Sci. 123, 3256-3265 (2010).

25. Katz, Y. et al. Musashi proteins are post-transcriptional regulators of the epithelial-luminal cell state. eLife 3, e03915 (2014).

26. Sakakibara, S. et al. RNA-binding protein Musashi family: roles for CNS stem cells and a subpopulation of ependymal cells revealed by targeted disruption and antisense ablation. Proc. Natl Acad. Sci. USA 99, 15194-15199 (2002).

27. Subramanian, A. et al. Gene set enrichment analysis: a knowledge-based approach for interpreting genome-wide expression profiles. Proc. Natl Acad. Sci. USA 102, 15545-15550 (2005).

28. Hirata, A. et al. Dose-dependent roles for canonical Wnt signalling in de novo crypt formation and cell cycle properties of the colonic epithelium. Development 140, 66-75 (2013).

29. Imai, T. et al. The neural RNA-binding protein Musashil translationally regulates mammalian numb gene expression by interacting with its mRNA. Mol. Cell Biol. 21, 3888-3900 (2001).

30. Battelli, C., Nikopoulos, G. N., Mitchell, J. G. \& Verdi, J. M. The RNA-binding protein Musashi-1 regulates neural development through the translational repression of p21WAF-1. Mol. Cell Neurosci. 31, 85-96 (2006).

31. Powell, A. E. et al. The pan-ErbB negative regulator Lrig1 is an intestinal stem cell marker that functions as a tumor suppressor. Cell 149, 146-158 (2012).

32. Howe, J. R. et al. Germline mutations of the gene encoding bone morphogenetic protein receptor $1 \mathrm{~A}$ in juvenile polyposis. Nat. Genet. 28, 184-187 (2001).
33. He, X. C. et al. PTEN-deficient intestinal stem cells initiate intestinal polyposis. Nat. Genet. 39, 189-198 (2007).

34. Goel, A. et al. Frequent inactivation of PTEN by promoter hypermethylation in microsatellite instability-high sporadic colorectal cancers. Cancer Res. 64, 3014-3021 (2004).

35. Naguib, A. et al. Alterations in PTEN and PIK3CA in colorectal cancers in the EPIC Norfolk study: associations with clinicopathological and dietary factors. BMC Cancer 11, 123 (2011).

36. Marsh, V. et al. Epithelial Pten is dispensable for intestinal homeostasis but suppresses adenoma development and progression after Apc mutation. Nat. Genet. 40, 1436-1444 (2008).

37. Stocker, H. et al. Living with lethal PIP3 levels: viability of flies lacking PTEN restored by a PH domain mutation in Akt/PKB. Science 295, 2088-2091 (2002).

38. Ashton, G. H. et al. Focal adhesion kinase is required for intestinal regeneration and tumorigenesis downstream of Wnt/c-Myc signaling. Dev. Cell 19, 259-269 (2010).

39. Fujishita, T., Aoki, K., Lane, H. A., Aoki, M. \& Taketo, M. M. Inhibition of the mTORC1 pathway suppresses intestinal polyp formation and reduces mortality in ApcDelta716 mice. Proc. Natl Acad. Sci. USA 105, $13544-13549$ (2008).

40. Elyada, E. et al. CKIalpha ablation highlights a critical role for p53 in invasiveness control. Nature 470, 409-413 (2011).

41. Spears, E. \& Neufeld, K. L. Novel double-negative feedback loop between adenomatous polyposis coli and Musashil in colon epithelia. J. Biol. Chem. 286, 4946-4950 (2011).

42. Wang, X. Y. et al. Musashil modulates mammary progenitor cell expansion through proliferin-mediated activation of the Wnt and Notch pathways. Mol. Cell Biol. 28, 3589-3599 (2008).

43. Beard, C., Hochedlinger, K., Plath, K., Wutz, A. \& Jaenisch, R. Efficient method to generate single-copy transgenic mice by site-specific integration in embryonic stem cells. Genesis 44, 23-28 (2006).

44. Shibata, H. et al. Rapid colorectal adenoma formation initiated by conditional targeting of the Apc gene. Science 278, 120-123 (1997).

45. Sato, T. et al. Single Lgr 5 stem cells build crypt-villus structures in vitro without a mesenchymal niche. Nature 459, 262-265 (2009).

46. Gracz, A. D., Puthoff, B. J. \& Magness, S. T. Identification, isolation, and culture of intestinal epithelial stem cells from murine intestine. Methods Mol. Biol. 879, 89-107 (2012).

47. Chi, S. W., Zang, J. B., Mele, A. \& Darnell, R. B. Argonaute HITS-CLIP decodes microRNA-mRNA interaction maps. Nature 460, 479-486 (2009).

48. Trapnell, C., Pachter, L. \& Salzberg, S. L. TopHat: discovering splice junctions with RNA-Seq. Bioinformatics 25, 1105-1111 (2009).

49. Uren, P. J. et al. Site identification in high-throughput RNA-protein interaction data. Bioinformatics 28, 3013-3020 (2012).

50. Bailey, T. L. et al. MEME SUITE: tools for motif discovery and searching. Nucleic Acids Res. 37, W202-W208 (2009).

51. Rhodes, D. R. et al. Oncomine 3.0: genes, pathways, and networks in a collection of 18,000 cancer gene expression profiles. Neoplasia 9, 166-180 (2007).

\section{Acknowledgements}

We acknowledge members of the University of Pennsylvania P01 Center for Molecular Studies in Digestive and Liver Diseases for technical support and thoughtful discussions, particularly the Drs Anil Rustgi and John Lynch and members of their laboratories, as well as the Center's Molecular Pathology and Imaging core for histological processing, microscopy and analysis. G.M. was supported by Mr William H. and Mrs Alice Goodwin and the Commonwealth Foundation for Cancer Research and The Experimental Therapeutics Center of Memorial Sloan-Kettering Cancer Center. M.Y. was supported by a Howard Hughes Medical Institute International Student Fellowship. M.G.K. was supported by the US National Institutes of Health National Institute of Diabetes and Digestive and Kidney Diseases Career Development Award and NIDDK NIH R01-DK101989-01A1, Louis V Gerstner Young Investigator Award and the American Society of Hematology Junior Scholar Award, Kimmel Scholar Award and V-Scholar Award. Z.Y. was supported by the National Natural Science Foundation of China (NSFC, 31271584), the National Basic Research programme of China (973 programme, 2011CB944103) and the National Transgenic Breeding Project of China (2011ZX08009-001-003) and 2015SKLAB6-16. C.J.L. was supported by a pilot award from the Penn P01 Center for Molecular Studies in Digestive and Liver Diseases, a grant from the State of Pennsylvania Health Research Formula Fund, a Fellowship from the W.W. Smith Charitable Trust and R01 CA16865 from the NCI. This work was supported in part by the NIH/NIDDK Center for Molecular Studies in Digestive and Liver Diseases (P30DK050306) and its core facilities.

\section{Author contributions}

C.J.L. and M.G.K. designed and developed the Msi mouse models used in the study. S.W., N.L., Z.Y. and C.J.L. designed and performed all experiments unless noted otherwise below. M.Y. performed mTOR and PIP3 analyses, irradiation and stem cell flow cytometry, A.N.-D. and K.P. performed mouse husbandry, genotype analyses and technical support. F.L. and B.D.G. performed analysis of CLIP-Seq data. S.R. performed gene expression analyses. G.M. performed electrophoretic mobility shift assays. Y.K. performed TCGA data analysis. C.J.L. and S.W. wrote the manuscript with editorial support from N.L., Z.Y. and M.G.K. 


\section{Additional information}

Accession codes: CLIP-Seq data are publicly available in the NCBI GEO repository, accession number GSE64388. Transcriptome profiling data are publicly available in the NCBI GEO repository, accession number GSE64643.

Supplementary Information accompanies this paper at http://www.nature.com/ naturecommunications
Competing financial interests: The authors declare no competing financial interests.

Reprints and permission information is available online at http://npg.nature.com/ reprintsandpermissions/

How to cite this article: Wang, S. et al. Transformation of the intestinal epithelium by the MSI2 RNA-binding protein. Nat. Commun. 6:6517 doi: 10.1038/ncomms7517 (2015). 